\title{
Article \\ Diverse Multiple Lump Analytical Solutions for Ion Sound and Langmuir Waves
}

\author{
Abdulmohsen D. Alruwaili ${ }^{1}$, Aly R. Seadawy ${ }^{2, *}$, Syed T. R. Rizvi ${ }^{3}$ and Sid Ahmed O. Beinane ${ }^{1}$ \\ 1 Mathematics Department, College of Science, Jouf University, Sakaka 72341, Saudi Arabia; \\ adalruwail@ju.edu.sa (A.D.A.); sabeinane@ju.edu.sa (S.A.O.B.) \\ 2 Mathematics Department, Faculty of Science, Taibah University, \\ Al-Madinah Al-Munawarah 41411, Saudi Arabia \\ 3 Department of Mathematics, COMSATS University Islamabad, Lahore Campus, Sakaka 72341, Pakistan; \\ strrizvi@gmail.com \\ * Correspondence: aabdelalim@taibahu.edu.sa
}

check for

updates

Citation: Alruwaili, A.D.; Seadawy, A.R.; Rizvi, S.T.R.; Beinane, S.A.O. Diverse Multiple Lump Analytical Solutions for Ion Sound and Langmuir Waves. Mathematics 2022, 10, 200. https://doi.org/ $10.3390 /$ math10020200

Academic Editors: Nikolai A.

Kudryashov, Andrea Scapellato and Alberto Cabada

Received: 28 October 2021 Accepted: 23 December 2021 Published: 10 January 2022

Publisher's Note: MDPI stays neutral with regard to jurisdictional claims in published maps and institutional affiliations.

Copyright: (c) 2022 by the authors. Licensee MDPI, Basel, Switzerland. This article is an open access article distributed under the terms and conditions of the Creative Commons Attribution (CC BY) license (https:/ / creativecommons.org/licenses/by/ $4.0 /)$.

\begin{abstract}
In this work, we study a time-fractional ion sound and Langmuir waves system (FISLWS) with Atangana-Baleanu derivative (ABD). We use a fractional ABD operator to transform our system into an ODE. We investigate multiwaves, periodic cross-kink, rational, and interaction solutions by the combination of rational, trigonometric, and various bilinear functions. Furthermore, 3D, 2D, and relevant contour plots are presented for the natural evolution of the gained solutions under the selection of proper parameters.
\end{abstract}

Keywords: multiwave; periodic cross-kink solutions; rational and interaction solutions; timefractional ion sound and Langmuir waves system

\section{Introduction}

At the present time, various real phenomena have been formulated by integer-order nonlinear partial differential equations (NPDEs). These supermodels are studied in different domains of sciences, such as engineering, chemistry, biology, physics, optics, etc. However, it is not enough to use integer order where the nonlocal property does not appear in these forms, so different models have been systematized in fractional NPDEs to determine that kind of similarity [1]. By using numerical and computational schemes, these models give more familiar properties [2-10]. To use most of these schemes, one needs fractional operator to transform the fractional forms into nonlinear ODEs with integer orders such as conformable fractional derivative, Caputo, Caputo-Fabrizio definition, Riemann-Liouville derivatives, and so on [11-24]. These operators have been applied to estimate the numeric and exact solutions of fractional order NPDEs through different integration schemes, such as $\left(\phi^{6}\right)$-model expansion [25], $\left(\frac{G^{\prime}}{G}\right)$-expansion [26], $\tan \left(\frac{\Phi(\rho)}{2}\right)$ expansion [27], Kudryashove scheme [28], $\exp \left(\left(-\frac{\Psi^{\prime}}{\Psi}\right) \eta\right)$-expansion [29], extended auxiliary equation technique [30], and so many others.

Here, we consider the FISLWS as follows [17],

$$
\begin{aligned}
& i^{A B} D_{t}^{\alpha} m+\frac{1}{2} m_{x x}-n m=0, \\
& { }^{A B} D_{t}^{2 \alpha} n-n_{x x}-2\left(|m|^{2}\right)_{x x}=0, \quad t>0, \quad 0<\alpha \leq 1 .
\end{aligned}
$$

where $m e^{-i \omega_{p} t}$ and $n$ illustrate the normalized electric-field of the Langmuir oscillation and perturbation of density, respectively. Both $x$ and $t$ are normalized variables and ${ }^{A B} D_{t}^{\alpha}$ is the $\mathrm{AB}$ fractional operator in $t$ direction. 
ABD operator is well defined as

$$
{ }^{A B D} D_{a^{+}}^{\alpha} F(t)=\frac{B(\alpha)}{1-\alpha} \frac{d}{d t} \int_{a}^{t} F(x) G_{\alpha}\left(\frac{-\alpha(t-\alpha)^{\alpha}}{1-\alpha}\right) d x,
$$

where $G_{\alpha}$ is Mittag-Leffler function, defined as

$$
G_{\alpha}\left(\frac{-\alpha(t-\alpha)^{\alpha}}{1-\alpha}\right)=\sum_{n=0}^{\infty} \frac{\left(\frac{-\alpha}{1-\alpha}\right)^{s}(t-x)^{\alpha s}}{\Gamma(\alpha s+1)},
$$

and $B(\alpha)$ is the normalization function that satisfies $B(1)=B(0)=1$. Thus,

$$
{ }^{A B D} D_{a^{+}}^{\alpha} F(t)=\frac{B(\alpha)}{1-\alpha} \sum_{n=0}^{\infty}\left(\frac{-\alpha}{1-\alpha}\right)^{s} R L I_{a}^{\alpha s} F(t) .
$$

for more properties of this operator. This leads towards the following form,

$$
\begin{aligned}
& m(x, t)=u(\xi) e^{i \vartheta}, \quad n(x, t)=v(\xi), \\
& \vartheta=a x+\frac{\beta(1-\alpha) t^{-s}}{B(\alpha) \sum_{s=0}^{\infty}\left(-\frac{\alpha}{1-\alpha}\right)^{s} \Gamma(1-\alpha s)}, \\
& \xi=b x+\frac{\gamma(1-\alpha) t^{-s}}{B(\alpha) \sum_{s=0}^{\infty}\left(-\frac{\alpha}{1-\alpha}\right)^{s} \Gamma(1-\alpha s)},
\end{aligned}
$$

where $\beta$ and $\gamma$ are arbitrary constants. This wave alteration converts Equation (1) into the following ODE.

$$
\begin{aligned}
\frac{1}{2} b^{2} u^{\prime \prime}+i(\gamma+a b) u^{\prime}-\frac{1}{2}\left(a^{2}+2 \beta\right) u-u v & =0 \\
\left(\gamma^{2}-b^{2}\right) v^{\prime \prime}-4 b^{2}\left(u^{\prime 2}+u u^{\prime \prime}\right) & =0 .
\end{aligned}
$$

Here, $u$ and $v$ are the functions of $\xi$. By separating the Img part from the first part of Equation (6),

$$
\gamma+a b=0 \Longrightarrow \gamma=-a b \text {. }
$$

and then by integrating the second part of Equation (6) by two times the w.r.t $\xi$, we obtain

$$
v=\frac{2 b^{2}}{-b^{2}+\gamma^{2}} u^{2}=\frac{2}{a^{2}-1} u^{2} .
$$

Equations (7) and (8) transform Equation (6) into the following form:

$$
\begin{aligned}
& u^{\prime \prime}-\frac{4}{b^{2}\left(a^{2}-1\right)} u^{3}-\frac{a^{2}+2 \beta}{b^{2}} u=0, \\
& \text { or } \\
& u^{\prime \prime}=\frac{4}{b^{2}\left(a^{2}-1\right)} u^{3}+\frac{a^{2}+2 \beta}{b^{2}} u .
\end{aligned}
$$

The contents of this paper are arranged as follows: In Section 2, we present M-shaped rational solitons. In Section 3, we evaluate M-shaped interaction solutions. In Section 4, we find the multiwaves solution. In Section 5, we study homoclinic breather. In Section 6, we investigate periodic cross-kink solutions. In Section 7, we present results and discussions and Section 8 contains concluding remarks. 


\section{M-Shaped Rational Solitons}

By using the following log transformation,

$$
u=u_{0}+2(\ln \Phi)_{\xi} .
$$

Equation (10) transforms Equation (9) into the following bilinear form:

$$
\begin{gathered}
u_{0}\left(-a^{4}-4 u_{0}^{2}+a^{2}(1-2 \beta)+2 \beta\right) \Phi^{3}+4\left(-8+\left(-1+a^{2}\right) b^{2}\right) \Phi^{\prime 3}-6 \Phi \Phi^{\prime}\left(8 u_{0} \Phi^{\prime}+\right. \\
\left.\left(-1+a^{2}\right) b^{2} \Phi^{\prime \prime}\right)-2 \Phi^{2}\left(\left(a^{4}+12 u_{0}^{2}-2 \beta+a^{2}(-1+2 \beta)\right) \Phi^{\prime}-\left(-1+a^{2}\right) b^{2} \Phi^{\prime \prime \prime}\right)=0 .
\end{gathered}
$$

We choose M-shaped rational solution in bilinear form for $\Phi$, as follows [31]:

$$
\Phi=\left(b_{2}+b_{1} \xi\right)^{2}+\left(b_{4}+b_{3} \xi\right)^{2}+b_{5}
$$

where $b_{i}(1 \leq i \leq 5)$ all are real-valued parameters to be measured. Inserting $\Phi$ into Equation (11) and collecting all powers of $\xi$, we obtain proper results, as follows (See Figures 1 and 2):

Set I. For $b_{2}=0$,

$$
a=a, b=b, \beta=-\frac{a^{4}-a^{2}+4 u_{0}^{2}}{2\left(a^{2}-1\right)}, b_{1}=i b_{3}, b_{3}=b_{3}, b_{4}=b_{4}, b_{5}=b_{5}, u_{0}=u_{0} .
$$

Using this in Equation (12), and then by using Equations (8) and (10), we obtain

$$
\begin{gathered}
u(\xi)=u_{0}+\frac{2\left(-2 b_{3}^{2} \xi+2 b_{3}\left(b_{4}+b_{3} \xi\right)\right)}{b_{5}-b_{3}^{2} \xi^{2}+\left(b_{4}+b_{3} \xi\right)^{2}} \\
v(\xi)=\frac{2}{a^{2}-1}\left(u_{0}+\frac{2\left(-2 b_{3}^{2} \zeta+2 b_{3}\left(b_{4}+b_{3} \xi\right)\right)}{b_{5}-b_{3}^{2} \xi^{2}+\left(b_{4}+b_{3} \xi\right)^{2}}\right)^{2} .
\end{gathered}
$$

To obtain final results, we use Equation (5):

$$
\begin{aligned}
& m_{21}(x, t)=e^{i\left(a x-\frac{t^{-s}\left(a^{4}-a^{2}+4 u_{0}^{2}\right)(1-\alpha)}{\left.2\left(a^{2}-1\right) B(\alpha) \sum_{s=0}^{\infty}\left(-\frac{\alpha}{1-\alpha}\right)^{s} \Gamma(1-\alpha)\right)}\right)}\left(u_{0}+\frac{2\left(2 b_{3}\left(b_{4}+b_{3} \Omega\right)-2 b_{3}^{2} \Omega\right)}{b_{5}+\left(b_{4}+b_{3} \Omega\right)^{2}-b_{3} \Omega^{2}}\right), \\
& n_{22}(x, t)=\frac{2}{\left(a^{2}-1\right)}\left(u_{0}+\frac{2\left(2 b_{3}\left(b_{4}+b_{3} \Omega\right)-2 b_{3}^{2} \Omega\right)}{b_{5}+\left(b_{4}+b_{3} \Omega\right)^{2}-b_{3} \Omega^{2}}\right)^{2},
\end{aligned}
$$

where $\Omega=\left(b x-\frac{a b t^{-s}(1-\alpha)}{B(\alpha) \sum_{s=0}^{\infty}\left(-\frac{\alpha}{1-\alpha}\right)^{S} \Gamma(1-\alpha s)}\right)$.

Set II. For $b_{5}=0$,

$$
a=a, b=b, \beta=-\frac{a^{4}-a^{2}+4 u_{0}^{2}}{2(a-1)(a+1)}, b_{1}=i b_{3}, b_{2}=b_{2}, b_{3}=b_{3}, b_{4}=b_{4}, u_{0}=u_{0} .
$$

Using this in Equation (12), and then by using Equations (8) and (10) in Equation (5), we obtain

$$
\begin{gathered}
m_{23}(x, t)=e^{i\left(a x-\frac{t^{-s}\left(a^{4}-a^{2}+4 u^{2}\right)(1-\alpha)}{2\left(a^{2}-1\right) B(\alpha) \sum_{s=0}^{\infty}\left(-\frac{\alpha}{1-\alpha}\right)^{s} \Gamma(1-\alpha s)}\right)}\left(u_{0}+\frac{2\left(2 i b_{3}\left(b_{2}+i b_{3} \Omega\right)+2 b_{3}\left(b_{4}+b_{3} \Omega\right)\right)}{\left(b_{2}+i b_{3} \Omega\right)^{2}+\left(b_{4}+b_{3} \Omega\right)^{2}}\right), \\
n_{24}(x, t)=\frac{2}{\left(a^{2}-1\right)}\left(u_{0}+\frac{2\left(2 i b_{3}\left(b_{2}+i b_{3} \Omega\right)+2 b_{3}\left(b_{4}+b_{3} \Omega\right)\right)}{\left(b_{2}+i b_{3} \Omega\right)^{2}+\left(b_{4}+b_{3} \Omega\right)^{2}}\right)^{2}, \\
\text { where } \Omega=\left(b x-\frac{a b t^{-s}(1-\alpha)}{B(\alpha) \sum_{s=0}^{\infty}\left(-\frac{\alpha}{1-\alpha}\right)^{s} \Gamma(1-\alpha s)}\right) .
\end{gathered}
$$




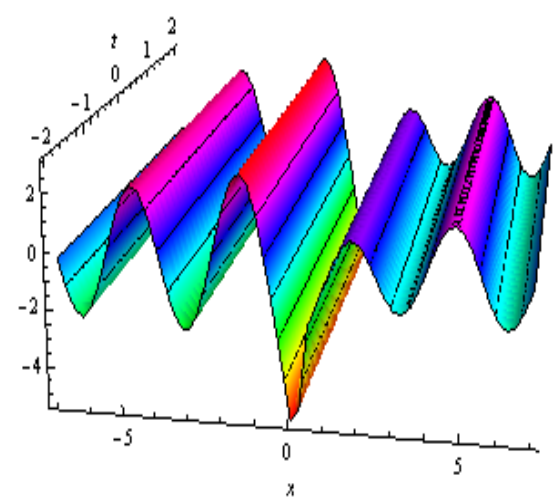

(a)

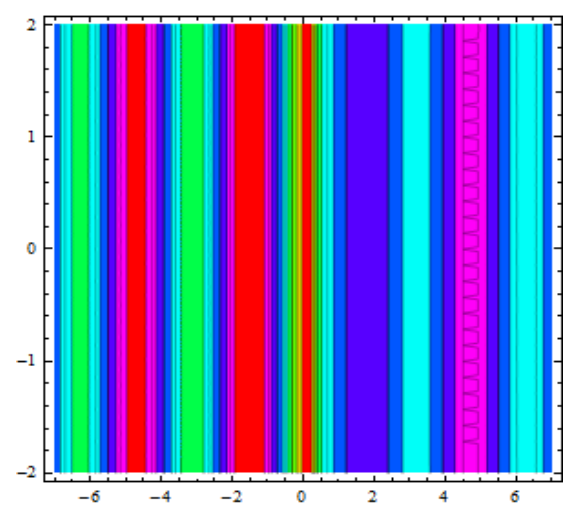

(b)

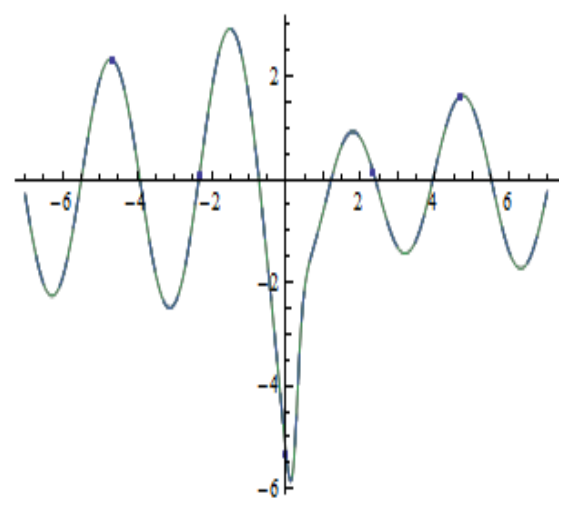

(c)

Figure 1. Plots of $m_{23}(x, t)$ in Equation (17) for $a=2, b=1.2, u_{0}=-2, b_{2}=3, b_{3}=5, b_{4}=-3$, $\alpha=0.9$, respectively as three-dimensions in (a); contour in (b) and two-dimensions in (c)

Set III. For $u_{0}=0$,

$$
a=a, b=b, \beta=-\frac{1}{2} a^{2}, b_{1}=i b_{3}, b_{2}=b_{2}, b_{3}=b_{3}, b_{4}=b_{4}, b_{5}=b_{5} .
$$

Using this in Equation (12), and then by using Equations (8) and (10), we obtain

$$
\begin{gathered}
u(\xi)=\frac{2\left(2 i b_{3}\left(b_{2}+i b_{3} \xi\right)+2 b_{3}\left(b_{4}+b_{3} \xi\right)\right)}{b_{5}+\left(b_{2}+i b_{3} \xi\right)^{2}+\left(b_{4}+b_{3} \xi\right)^{2}}, \\
v(\xi)=\frac{8\left(2 i b_{3}\left(b_{2}+i b_{3} \xi\right)+2 b_{3}\left(b_{4}+b_{3} \xi\right)\right)^{2}}{\left(-1+a^{2}\right)\left(b_{5}+\left(b_{2}+i b_{3} \xi\right)^{2}+\left(b_{4}+b_{3} \xi\right)^{2}\right)^{2}} .
\end{gathered}
$$

To obtain final results, we use Equation (5):

$$
\begin{aligned}
& m_{25}(x, t)=\frac{2 e^{i\left(a x-\frac{a^{2} t^{-s}(1-\alpha)}{2 B(\alpha) \sum_{s=0}^{\infty}\left(-\frac{\alpha}{1-\alpha}\right)^{s} \Gamma(1-\alpha)}\right)}\left(2 i b_{3}\left(b_{2}+i b_{3} \Omega\right)+2 b_{3}\left(b_{4}+b_{3} \Omega\right)\right)}{b_{5}+\left(b_{2}+i b_{3} \Omega\right)^{2}+\left(b_{4}+b_{3} \Omega\right)^{2}}, \\
& n_{26}(x, t)=\frac{8}{\left(a^{2}-1\right)}\left(\frac{2 i b_{3}\left(b_{2}+i b_{3} \Omega\right)+2 b_{3}\left(b_{4}+b_{3} \Omega\right)}{b_{5}+\left(b_{2}+i b_{3} \Omega\right)^{2}+\left(b_{4}+b_{3} \Omega\right)^{2}}\right)^{2},
\end{aligned}
$$

where $\Omega=\left(b x-\frac{a b t^{-s}(1-\alpha)}{B(\alpha) \sum_{s=0}^{\infty}\left(-\frac{\alpha}{1-\alpha}\right)^{s} \Gamma(1-\alpha s)}\right)$.

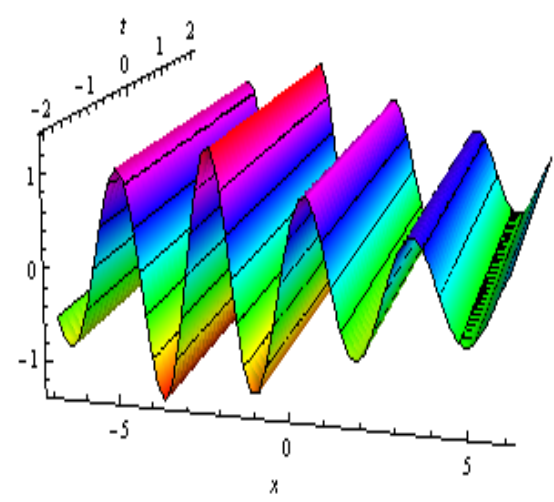

(a)

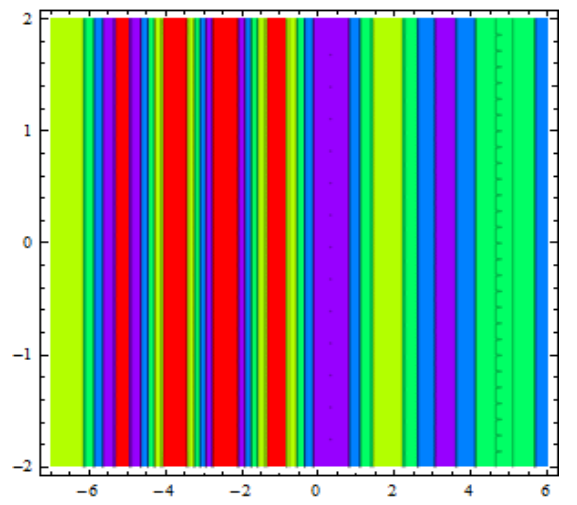

(b)

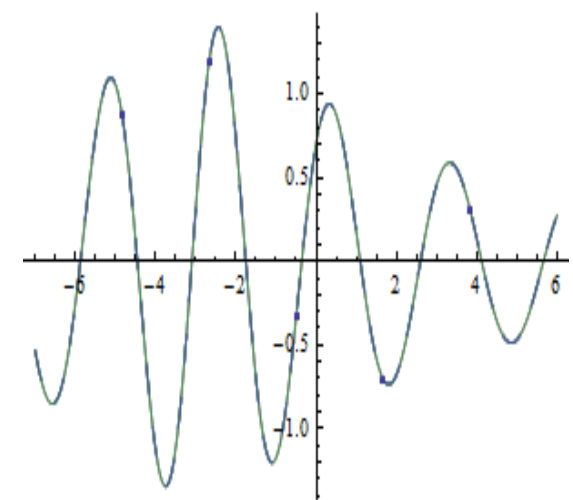

(c)

Figure 2. represented three-dimensions in (a); contour in (b) and two-dimensions in (c), Plots of $m_{25}(x, t)$ in Equation (20) for $a=2, b=0.5, b_{2}=-3, b_{3}=1, b_{4}=3, b_{5}=-1$, $\alpha=0.8$, respectively. 


\section{M-Shaped Rational Soliton Interactions with}

In this part, we evaluate M-shaped rational interactions with periodic and kink waves by using exponential and cos function in bilinear combinations.

\subsection{One-Kink Soliton}

For this, the bilinear form for $\Phi$ is as follows [31]:

$$
\Phi=\left(b_{2}+b_{1} \xi\right)^{2}+\left(b_{4}+b_{3} \xi\right)^{2}+c e^{b_{6}+b_{5} \xi}+b_{7},
$$

where $b_{i}(1 \leq i \leq 7)$, all are real-valued parameters to be measured. Inserting $\Phi$ into Equation (11) and collecting all powers of $e^{b_{5} \tau+b_{6}}, e^{2\left(b_{5} \tau+b_{6}\right)}, e^{3\left(b_{5} \tilde{\xi}+b_{6}\right)}, \xi e^{b_{5} \xi+b_{6}}, \xi e^{2\left(b_{5} \tau+b_{6}\right)}$, $\xi^{2} e^{b_{5} \xi+b_{6}}, \xi^{3} e^{b_{5} \xi+b_{6}}, \xi^{4} e^{b_{5} \xi+b_{6}}$, and $\xi$, we obtain proper results, as follows (See Figures 3-6):

Set I. For $b_{2}=b_{6}=0$,

$$
\begin{gathered}
a=a, b=2 \sqrt{2} \sqrt{\frac{1}{a^{2}-1}}, \beta=-\frac{a^{4}-a^{2}+4 b_{5}^{2}}{2(a-1)(a+1)}, b_{1}=i b_{3}, b_{3}=b_{3}, b_{4}=b_{4}, b_{5}=b_{5}, \\
b_{7}=b_{7}, u_{0}=-b_{5} .
\end{gathered}
$$

Using Equation (22) in Equation (21), and then by using Equations (8) and (10), we obtain

$$
\begin{gathered}
u(\xi)=-b_{5}+\frac{2\left(b_{5} c e^{b_{5} \xi}-2 b_{3}^{2} \xi+2 b_{3}\left(b_{4}+b_{3} \xi\right)\right)}{b_{7}+c e^{b_{5}} \xi-b_{3}^{2} \xi^{2}+\left(b_{4}+b_{3} \xi\right)^{2}} \\
v(\xi)=\frac{2\left(b_{5}\left(b_{4}^{2}+b_{7}-c e^{b_{5}}\right)+2 b_{3} b_{4}\left(-2+b_{5} \xi\right)\right)^{2}}{\left(-1+a^{2}\right)\left(b_{4}^{2}+b_{7}+c e^{b_{5}}+2 b_{3} b_{4} \xi\right)^{2}} .
\end{gathered}
$$

Using Equation (5) to obtain the required solution for Equation (1),

$$
\begin{gathered}
m_{31}(x, t)=-\frac{e^{\Delta}\left(4 \sqrt{2} a \sqrt{\frac{1}{a^{2}-1}} b_{3} b_{4} b_{5}(\alpha-1)+t^{s}\left(b_{5}\left(b_{4}^{2}+b_{7}-c e^{\Delta_{1}}\right)+4 b_{3} b_{4}\left(-1+\sqrt{2} \sqrt{\frac{1}{a^{2}-1}} b_{5} x\right)\right) \Xi\right)}{4 \sqrt{2} a \sqrt{\frac{1}{a^{2}-1}} b_{3} b_{4}(\alpha-1)+t^{s}\left(b_{4}^{2}+b_{7}+c e^{\Delta_{1}}+4 \sqrt{2} \sqrt{\frac{1}{a^{2}-1}} b_{3} b_{4} x\right) \Xi}, \\
n_{32}(x, t)=\frac{2}{\left(a^{2}-1\right)}\left(\frac{4 \sqrt{2} a \sqrt{\frac{1}{a^{2}-1}} b_{3} b_{4} b_{5}(\alpha-1)+t^{s}\left(b_{5}\left(b_{4}^{2}+b_{7}-c e^{\Delta_{1}}\right)+4 b_{3} b_{4}\left(-1+\sqrt{2} \sqrt{\frac{1}{a^{2}-1}} b_{5} x\right)\right) \Xi}{4 \sqrt{2} a \sqrt{\frac{1}{a^{2}-1}} b_{3} b_{4}(\alpha-1)+t^{s}\left(b_{4}^{2}+b_{7}+c e^{\left.\Delta_{1}+4 \sqrt{2} \sqrt{\frac{1}{a^{2}-1}} b_{3} b_{4} x\right) \Xi}\right)^{2},}\right. \\
\text { where } \Delta=\frac{1}{2} i\left(2 a x+\frac{\left(-a^{2}+a^{4}+4 b_{5}^{2}\right) t^{-s}(-1+\alpha)}{(-1+a)(1+a) B(\alpha) \sum_{s=0}^{\infty}\left(-\frac{\alpha}{1-\alpha}\right)^{s} \Gamma(1-\alpha s)}\right), \\
\Delta_{1}=2 \sqrt{2} \sqrt{\frac{1}{a^{2}-1}} b_{5}\left(x+\frac{a t^{-s}(-1+\alpha)}{B(\alpha) \sum_{s=0}^{\infty}\left(-\frac{\alpha}{1-\alpha}\right)^{s} \Gamma(1-\alpha s)}\right), \\
\text { and } \Xi=B(\alpha) \sum_{s=0}^{\infty}\left(-\frac{\alpha}{1-\alpha}\right)^{s} \Gamma(1-\alpha s) .
\end{gathered}
$$




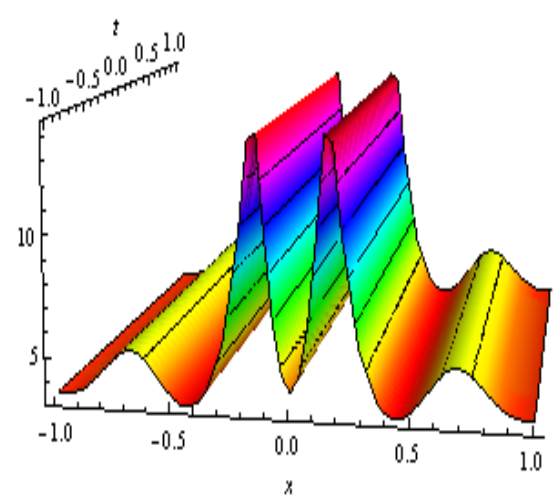

(a)

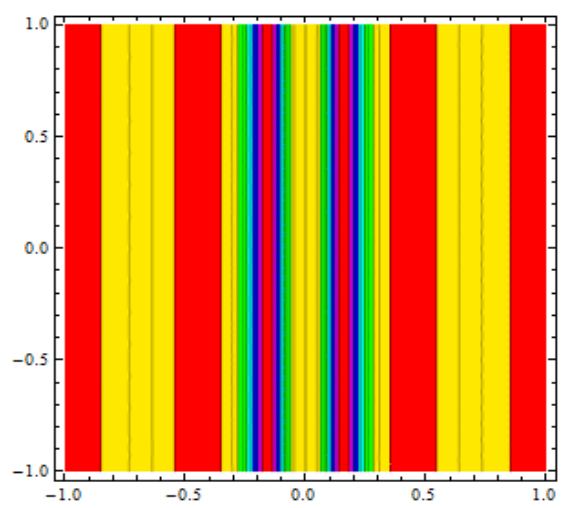

(b)

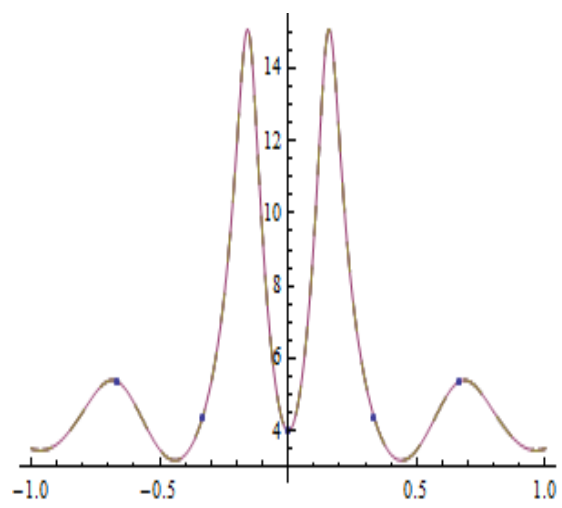

(c)

Figure 3. showed three-dimensions in (a); contour in (b) and two-dimensions in (c), Plots of $m_{31}(x, t)$ in Equation (24) for $a=0.2, b_{3}=1, b_{4}=2, b_{5}=-4, b_{7}=-3, c=1, \alpha=0.6$, respectively.

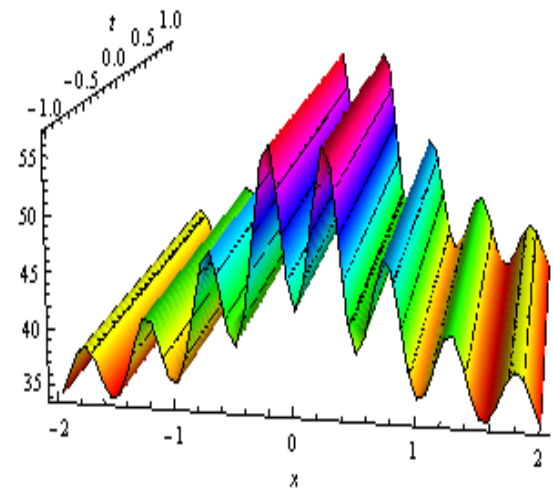

(a)

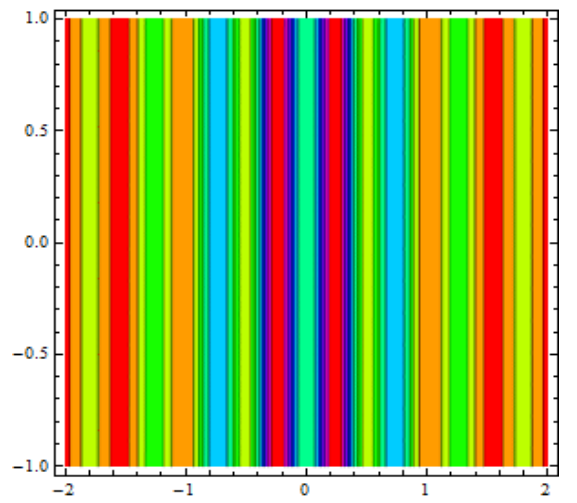

(b)

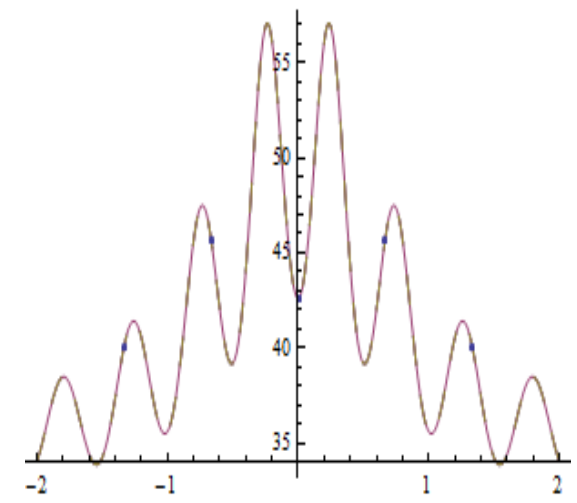

(c)

Figure 4. illustrated three-dimensions in (a); contour in (b) and two-dimensions in (c), Plots of $n_{32}(x, t)$ in Equation (24) for $a=0.2, b_{3}=1, b_{4}=5, b_{5}=-4, b_{7}=-3, c=1, \alpha=0.8$, respectively.

Set II. For $b_{1}=0$,

$a=a, b=0, \beta=-\frac{1}{2} a^{2}, b_{2}=b_{2}, b_{3}=b_{3}, b_{4}=-\frac{2 b_{3}}{u_{0}}, b_{5}=-\frac{1}{2} u_{0}, b_{6}=b_{6}, b_{7}=-\frac{b_{2}^{2} u_{0}^{2}-4 b_{3}^{2}}{u_{0}^{2}}, u_{0}=u_{0}$.

Using Equation (25) in Equation (21), and then by using Equations (8) and (10), we obtain

$$
\begin{gathered}
u(\xi)=u_{0}+\frac{2\left(-\frac{1}{2} c e^{b_{6}-\frac{u_{0} \xi}{2}} u_{0}+2 b_{3}\left(-\frac{2 b_{3}}{u_{0}}+b_{3} \xi\right)\right)}{b_{2}^{2}+c e^{b_{6}-\frac{u_{0} \xi}{2}}-\frac{b_{2}^{2} u_{0}^{2}-4 b_{3}^{2}}{u_{0}^{2}}+\left(-\frac{2 b_{3}}{u_{0}}+b_{3} \xi\right)^{2}}, \\
v(\xi)=\frac{2 b_{3}^{4} e^{u_{0} \xi} u_{0}^{6} \xi^{4}}{\left(-1+a^{2}\right)\left(c e^{b_{6}} u_{0}^{2}+b_{3}^{2} e^{\frac{u_{0} \xi}{2}}\left(8-4 u_{0} \xi+u_{0}^{2} \xi^{2}\right)\right)^{2}} .
\end{gathered}
$$

Using Equation (5), we obtain the required solution for Equation (1):

$$
\begin{array}{ll}
m_{33}(x, t) & =e^{i\left(a x-\frac{a^{2} t^{-s}(1-\alpha)}{2 B(\alpha) \sum_{s=0}^{\infty}\left(-\frac{\alpha}{1-\alpha}\right)^{s} \Gamma(1-\alpha)}\right)}\left(u_{0}+\frac{2\left(-\frac{4 b_{3}^{2}}{u_{0}}-\frac{1}{2} c e^{b_{6}} u_{0}\right)}{b_{2}^{2}+c e^{b_{6}}+\frac{4 b_{3}^{2}}{u_{0}^{2}}-\frac{b_{2}^{2} u_{0}^{2}-4 b_{3}^{2}}{u_{0}^{2}}}\right), \\
n_{34}(x, t) & =0, \quad(\because b=0) .
\end{array}
$$


Set III. For $b_{2}=b_{6}=0$,

$$
\begin{gathered}
a=a, b=\frac{2 \sqrt{-\frac{6 u_{0} b_{5}+4 u_{0}^{2}}{a^{2}-1}}}{b_{5}}, \beta=-\frac{4 u_{0}^{2}-a^{2}+a^{4}}{2\left(a^{2}-1\right)}, b_{1}=\frac{\frac{1}{3} i\left(b_{7}+b_{4}^{2}\right) u_{0}}{b_{4}}, b_{3}=-\frac{\left(b_{7}+b_{4}^{2}\right) u_{0}}{3 b_{4}}, \\
b_{4}=b_{4}, b_{5}=b_{5}, b_{7}=b_{7}, u_{0}=u_{0} .
\end{gathered}
$$

Using Equation (28) in Equation (21), and then by using Equations (8) and (10), we obtain

$$
\begin{gathered}
u(\xi)=u_{0}+\frac{2\left(b_{5} c e^{b_{5} \xi}-\frac{2\left(b_{4}^{2}+b_{7}\right)^{2} u_{0}^{2} \xi}{9 b_{4}^{2}}-\frac{2\left(b_{4}^{2}+b_{7}\right) u_{0}\left(b_{4}-\frac{\left(b_{7}+b_{4}^{2}\right) u_{0} \xi}{3 b_{4}}\right)}{3 b_{4}}\right)}{b_{7}+c e^{b_{5} \xi}-\frac{\left(b_{4}^{2}+b_{7}\right)^{2} u_{0}^{2} \xi^{2}}{9 b_{4}^{2}}+\left(b_{4}-\frac{\left(b_{7}+b_{4}^{2}\right) u_{0} \xi}{3 b_{4}}\right)^{2}} \\
v(\xi)=\frac{2\left(-6 b_{5} c e^{b_{5} \xi}+u_{0}\left(b_{7}-3 c e^{b_{5} \xi}+2 b_{7} u_{0} \xi+b_{4}^{2}\left(1+2 u_{0} \xi\right)\right)\right)^{2}}{\left(-1+a^{2}\right)\left(3 c e^{b_{5} \xi}+b_{4}^{2}\left(3-2 u_{0} \xi\right)+b_{7}\left(3-2 u_{0} \xi\right)\right)^{2}} .
\end{gathered}
$$

Now, using Equation (5), we obtain the required solution for Equation (1):

$$
\begin{gathered}
m_{35}(x, t)=\frac{e^{\Delta}\left(-4 \sqrt{2} a\left(b_{4}^{2}+b_{7}\right) u_{0}^{2} \Delta_{1}(\alpha-1)+t^{s}\left(6 b_{5}^{2} c e^{\Delta_{2}}-b_{5}\left(b_{4}^{2}+b_{7}-3 c e^{\Delta_{2}}\right) u_{0}-4 \sqrt{2}\left(b_{4}^{2}+b_{7}\right) u_{0}^{2} \Delta_{1} x\right) \Xi\right)}{-4 \sqrt{2} a\left(b_{4}^{2}+b_{7}\right) u_{0} \Delta_{1}(\alpha-1) t^{s}\left(3 b_{5}\left(b_{7}+c e^{\Delta_{2}}\right)-4 \sqrt{2} b_{7} u_{0} \Delta_{1} x+b_{4}^{2}\left(3 b_{5}-4 \sqrt{2} u_{0} \Delta_{1} x\right)\right) \Xi}, \\
n_{36}(x, t)=\frac{2}{\left(a^{2}-1\right)}\left(\frac{-4 \sqrt{2} a\left(b_{4}^{2}+b_{7}\right) u_{0}^{2} \Delta_{1}(\alpha-1)+t^{s}\left(6 b_{5}^{2} c e^{\Delta_{2}}-b_{5}\left(b_{4}^{2}+b_{7}-3 c e^{\Delta_{2}}\right) u_{0}-4 \sqrt{2}\left(b_{4}^{2}+b_{7}\right) u_{0}^{2} \Delta_{1} x\right) \Xi}{-4 \sqrt{2} a\left(b_{4}^{2}+b_{7}\right) u_{0} \Delta_{1}(\alpha-1) t^{s}\left(3 b_{5}\left(b_{7}+c e^{\Delta_{2}}\right)-4 \sqrt{2} b_{7} u_{0} \Delta_{1} x+b_{4}^{2}\left(3 b_{5}-4 \sqrt{2} u_{0} \Delta_{1} x\right)\right) \Xi}\right), \\
\text { where } \Delta=\frac{1}{2} i\left(2 a x+\frac{t^{-s}\left(-a^{2}+a^{4}+4 u_{0}^{2}\right)(-1+\alpha)}{(-1+a)(1+a) B(\alpha) \sum_{s=0}^{\infty}\left(-\frac{\alpha}{1-\alpha}\right)^{s} \Gamma(1-\alpha s)}\right), \Delta_{1}=\sqrt{-\frac{u_{0}\left(3 b_{5}+2 u_{0}\right)}{-1+a^{2}}}, \\
\Delta_{2}=\frac{2 \sqrt{2} t^{-s} \sqrt{-\frac{u_{0}\left(3 b_{5}+2 u_{0}\right)}{-1+a^{2}}}\left(a(-1+\alpha)+t^{s} x B(\alpha) \sum_{s=0}^{\infty}\left(-\frac{\alpha}{1-\alpha}\right)^{s} \Gamma(1-\alpha s)\right)}{B(\alpha) \sum_{s=0}^{\infty}\left(-\frac{\alpha}{1-\alpha}\right)^{s} \Gamma(1-\alpha s)}, \\
\text { and } \Xi=B(\alpha) \sum_{s=0}^{\infty}\left(-\frac{\alpha}{1-\alpha}\right)^{s} \Gamma(1-\alpha s) .
\end{gathered}
$$

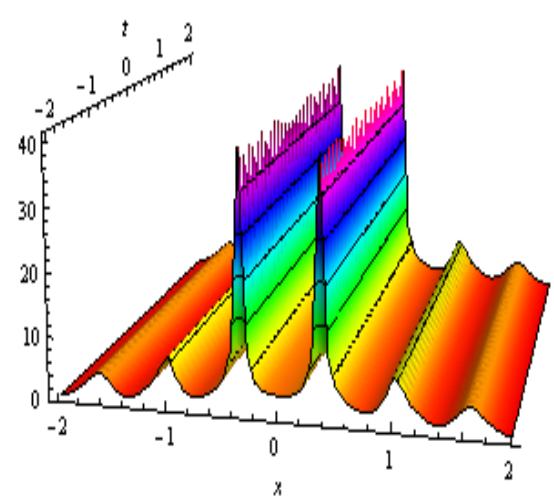

(a)

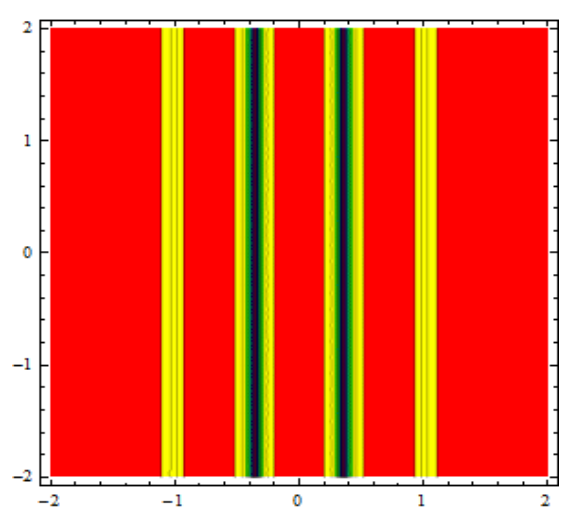

(b)

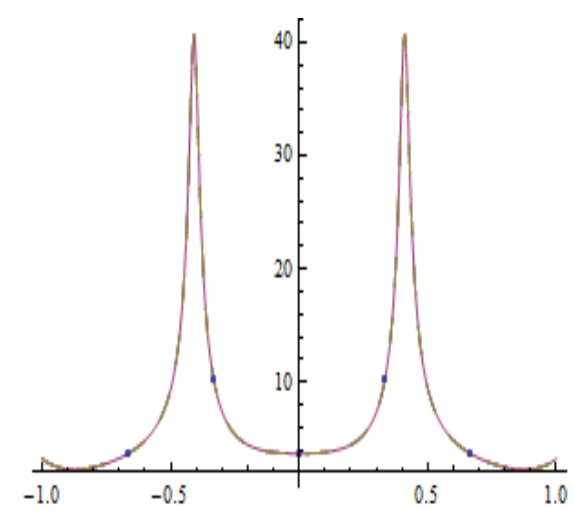

(c)

Figure 5. clarify three-dimensions in (a); contour in (b) and two-dimensions in (c), Plots of $m_{35}(x, t)$ in Equation (30) for $a=0.5, u_{0}=1, b_{4}=2, b_{5}=-4, b_{7}=-3, c=1$, $\alpha=0.8$, respectively. 


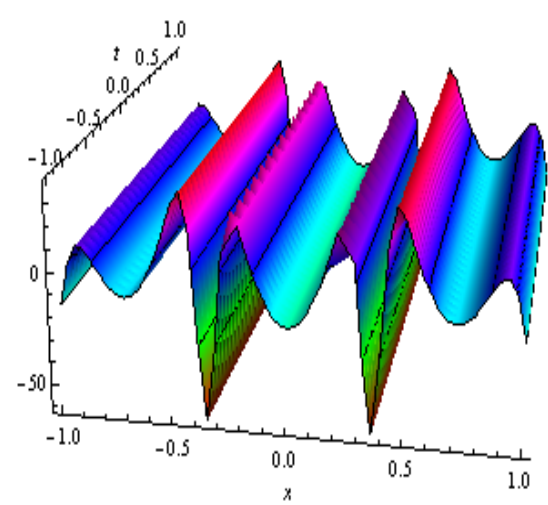

(a)

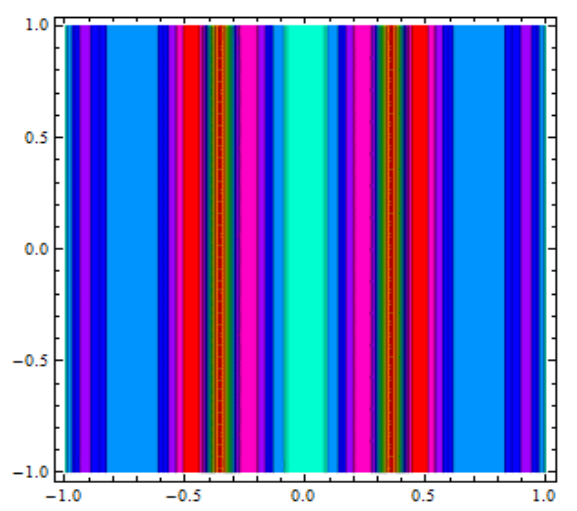

(b)

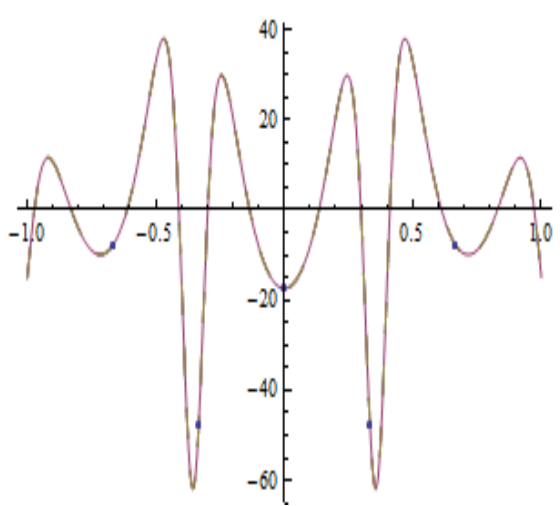

(c)

Figure 6. explain three-dimensions in (a); contour in (b) and two-dimensions in (c), Plots of $n_{36}(x, t)$ in Equation (30) for $a=0.3, u_{0}=0.8, b_{4}=2, b_{5}=-5, b_{7}=-3$, $c=0.4, \alpha=0.8$, respectively.

\subsection{Two-Kink Soliton}

For two-kink interaction, the bilinear solution for $\Phi$ is as follows (See Figures 7-9):

$$
\Phi=\left(b_{2}+b_{1} \xi\right)^{2}+\left(b_{4}+b_{3} \xi\right)^{2}+e^{b_{6}+b_{5} \xi}+e^{b_{8}+b_{7} \xi}+b_{9}
$$

where $b_{i}(1 \leq i \leq 9)$ and all are real-valued parameters to be found. Inserting $\Phi$ into Equation (11) and collecting all powers of $\xi$, and $e^{3\left(b_{5} \xi+b_{6}\right)}, e^{2\left(b_{5} \xi+b_{6}\right)}, e^{2\left(b_{7} \xi+b_{8}\right)}, \xi e^{b_{5} \xi+b_{6}}$, $\xi e^{2\left(b_{5} \xi+b_{6}\right)}, \xi e^{b_{7} \xi+b_{8}}, \xi e^{2\left(b_{7} \xi+b_{8}\right)}, \xi^{2} e^{b_{5} \xi+b_{6}}, \xi^{3} e^{b_{5} \xi+b_{6}}, \xi^{3} e^{b_{7} \xi+b_{8}}, \xi^{4} e^{b_{7} \xi+b_{8}}, \xi^{4} e^{b_{5} \xi+b_{6}}$, we obtain proper results, as follows:

Set I. For $u_{0}=0$,

$$
\begin{gathered}
a=a, b=2 \sqrt{2} \sqrt{\frac{1}{a^{2}-1}}, \beta=-\frac{a^{4}-a^{2}+16 b_{7}^{2}}{2(a-1)(a+1)}, b_{1}=i b_{3}, b_{2}=i b_{4}, b_{3}=b_{3}, b_{4}=b_{4}, \\
b_{5}=-b_{7}, b_{6}=b_{6}, b_{7}=b_{7}, b_{8}=b_{8}, b_{9}=b_{9} .
\end{gathered}
$$

Using Equation (32) in Equation (31), and then by using Equations (8) and (10), we obtain

$$
\begin{array}{r}
u(\xi)=\frac{2\left(-b_{7} e^{b_{6}-b_{7} \xi}+b_{7} e^{b_{8}+b_{7} \xi}+2 i b_{3}\left(i b_{4}+i b_{3} \xi\right)+2 b_{3}\left(b_{4}+b_{3} \xi\right)\right)}{b_{9}+e^{b_{6}-b_{7} \xi}+e^{b_{8}+b_{7} \xi}+\left(i b_{4}+i b_{3} \xi\right)^{2}+\left(b_{4}+b_{3} \xi\right)^{2}}, \\
v(\xi)=\frac{8}{-1+a^{2}}\left(\frac{-b_{7} e^{b_{6}-b_{7} \xi}+b_{7} e^{b_{8}+b_{7} \xi}+2 i b_{3}\left(i b_{4}+i b_{3} \xi\right)+2 b_{3}\left(b_{4}+b_{3} \xi\right)}{b_{9}+e^{b_{6}-b_{7}} \xi+e^{b_{8}+b_{7} \xi}+\left(i b_{4}+i b_{3} \xi\right)^{2}+\left(b_{4}+b_{3} \xi\right)^{2}}\right)^{2} .
\end{array}
$$

Using Equation (5), we obtain the required solution for Equation (1),

$$
\begin{aligned}
& m_{37}(x, t)=-\frac{2 b_{7} e^{\frac{1}{2} i\left(2 a x+\frac{\left(-a^{2}+a^{4}+16 b_{7}^{2}\right) t^{-s}(-1+\alpha)}{(-1+a)(1+a) B(\alpha) \sum_{s=0}^{\infty}\left(-\frac{\alpha}{1-\alpha}\right)^{S} \Gamma(1-\alpha s)}\right)}\left(e^{b_{6}}-e^{b_{8}+2 \Omega}\right)}{e^{b_{6}}+e^{b_{8}+2 \Omega}+b_{9} e^{\Omega}}, \\
& n_{38}(x, t)=\frac{8 b_{7}^{2}}{\left(a^{2}-1\right)}\left(\frac{e^{b_{6}}-e^{b_{8}+2 \Omega}}{e^{b_{6}}+e^{b_{8}+2 \Omega}+b_{9} e^{\Omega}}\right)^{2},
\end{aligned}
$$

where $\Omega=2 \sqrt{2} \sqrt{\frac{1}{a^{2}-1}} b_{7}\left(x+\frac{a t^{-s}(-1+\alpha)}{B(\alpha) \sum_{s=0}^{\infty}\left(-\frac{\alpha}{1-\alpha}\right)^{s} \Gamma(1-\alpha s)}\right)$. 


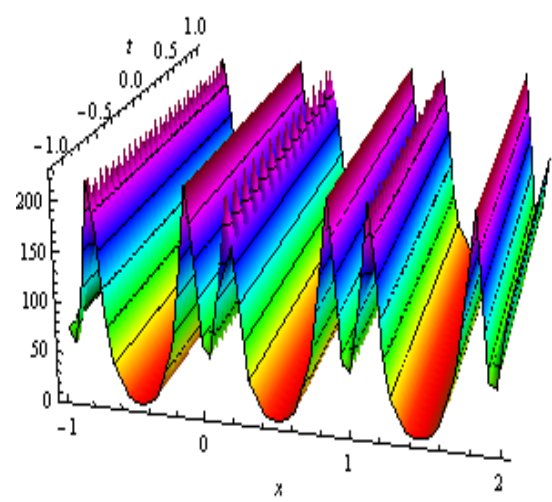

(a)

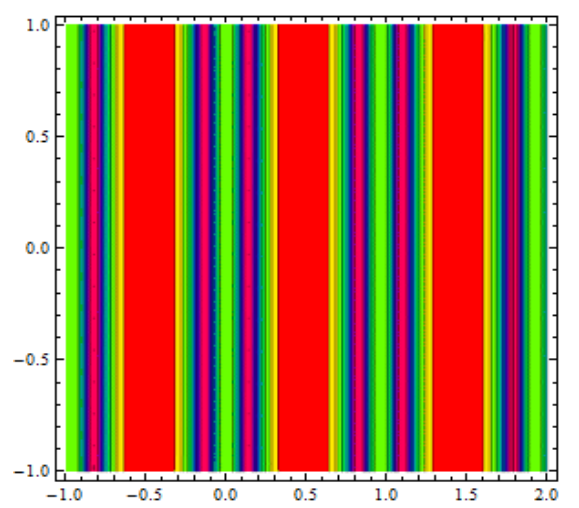

(b)

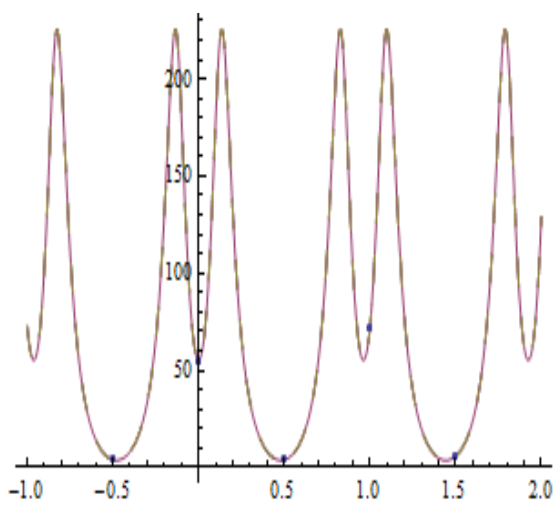

(c)

Figure 7. represented three-dimensions in (a); contour in (b) and two-dimensions in (c), Plots of $n_{38}(x, t)$ in Equation (34) for $a=0.5, b_{6}=1, b_{7}=-2, b_{8}=2, b_{9}=-6, \alpha=0.8$, respectively.

Set II.

$$
\begin{gathered}
a=a, b=4 \sqrt{\frac{2+i \sqrt{2}}{a^{2}-1}}, \beta=\frac{a^{2}-a^{4}-8 i(-2 i+\sqrt{2}) b_{7}^{2}}{2\left(a^{2}-1\right)}, b_{1}=i b_{3}, b_{2}=b_{2}, b_{3}=b_{3}, b_{4}=-i b_{2}, \\
b_{5}=\left(1+\frac{1}{2} i \sqrt{2}\right) b_{7}, b_{6}=b_{6}, b_{7}=b_{7}, b_{8}=b_{8}, b_{9}=0 .
\end{gathered}
$$

Using Equation (35) in Equation (31), and then by using Equations (8) and (10), we obtain

$$
\begin{array}{r}
u(\xi)=\frac{2\left(b_{7} e^{b_{8}+b_{7} \xi}+\left(1+\frac{i}{\sqrt{2}}\right) b_{7} e^{b_{6}+\left(1+\frac{i}{\sqrt{2}}\right) b_{7} \xi}+2 i b_{3}\left(b_{2}+i b_{3} \xi\right)+2 b_{3}\left(-i b_{2}+b_{3} \xi\right)\right)}{e^{b_{8}+b_{7} \xi}+e^{b_{6}+\left(1+\frac{i}{\sqrt{2}}\right) b_{7} \xi}+\left(b_{2}+i b_{3} \xi\right)^{2}+\left(-i b_{2}+b_{3} \xi\right)^{2}}, \\
v(\xi)=\frac{8}{-1+a^{2}}\left(\frac{b_{7} e^{b_{8}+b_{7} \xi}+\left(1+\frac{i}{\sqrt{2}}\right) b_{7} e^{b_{6}+\left(1+\frac{i}{\sqrt{2}}\right) b_{7} \xi}+2 i b_{3}\left(b_{2}+i b_{3} \xi\right)+2 b_{3}\left(-i b_{2}+b_{3} \xi\right)}{e^{b_{8}+b_{7} \xi}+e^{b_{6}+\left(1+\frac{i}{\sqrt{2}}\right) b_{7} \xi}+\left(b_{2}+i b_{3} \xi\right)^{2}+\left(-i b_{2}+b_{3} \xi\right)^{2}}\right)^{2} .
\end{array}
$$

Using Equation (5), we obtain the required solution for Equation (1):

$$
\begin{aligned}
& m_{39}(x, t)=\frac{b_{7} e^{\frac{1}{2} i\left(2 a x+\frac{\left(-a^{2}+a^{4}+8(2+i \sqrt{2}) b_{7}^{2}\right) t^{-s}(-1+\alpha)}{\left(-1+a^{2}\right) B(\alpha) \sum_{s=0}^{\infty}\left(-\frac{\alpha}{1-\alpha}\right)^{S} \Gamma(1-\alpha s)}\right.}\left(2 e^{b_{8}+\Omega}+(2+i \sqrt{2}) e^{b_{6}+\left(1+\frac{i}{\sqrt{2}}\right) \Omega}\right)}{e^{b_{8}+\Omega}+e^{b_{6}+\left(1+\frac{i}{\sqrt{2}}\right) \Omega}}, \\
& n_{40}(x, t)=\frac{2 b_{7}^{2}}{\left(a^{2}-1\right)}\left(\frac{2 e^{b_{8}+\Omega}+(2+i \sqrt{2}) e^{b_{6}+\left(1+\frac{i}{\sqrt{2}}\right) \Omega}}{e^{b_{8}+\Omega}+e^{b_{6}+\left(1+\frac{i}{\sqrt{2}}\right) \Omega}}\right)^{2}, \\
& \text { where } \Omega=4 \sqrt{\frac{2+i \sqrt{2}}{-1+a^{2}}} b_{7}\left(x+\frac{a t^{-s}(-1+\alpha)}{B(\alpha) \sum_{s=0}^{\infty}\left(-\frac{\alpha}{1-\alpha}\right)^{s} \Gamma(1-\alpha s)}\right) .
\end{aligned}
$$




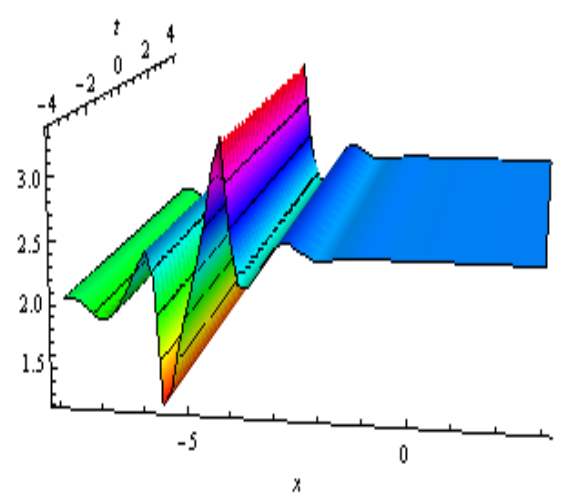

(a)

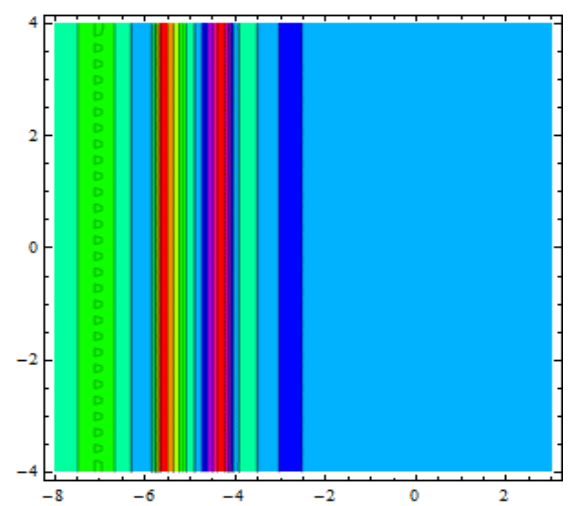

(b)

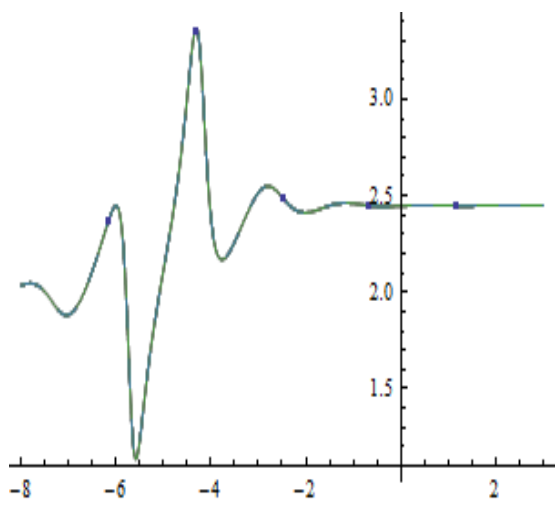

(c)

Figure 8. showed three-dimensions in (a); contour in (b) and two-dimensions in (c), Plots of $m_{39}(x, t)$ in Equation (37) for $a=1.5, b_{6}=8, b_{7}=-1, b_{8}=2, \alpha=0.6$, respectively.

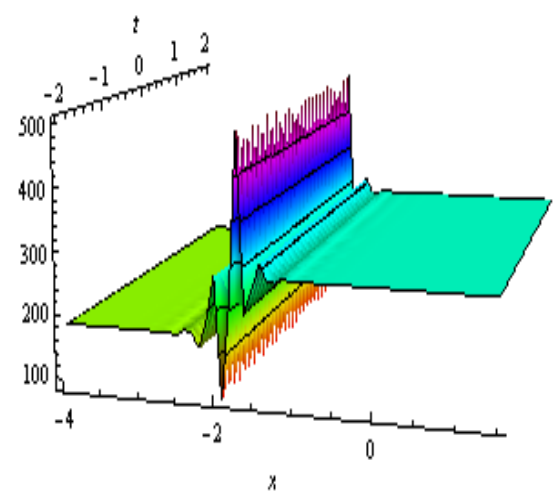

(a)

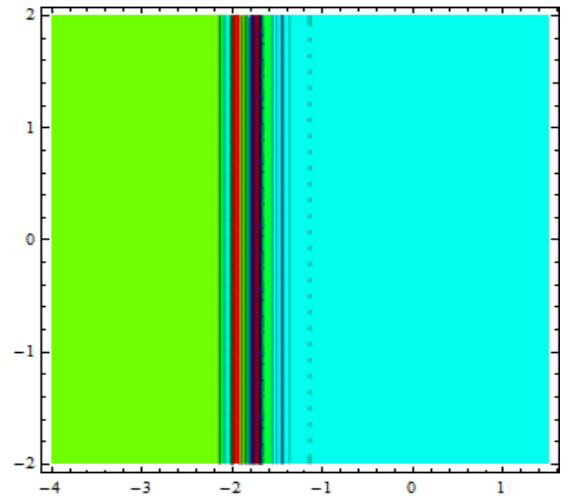

(b)

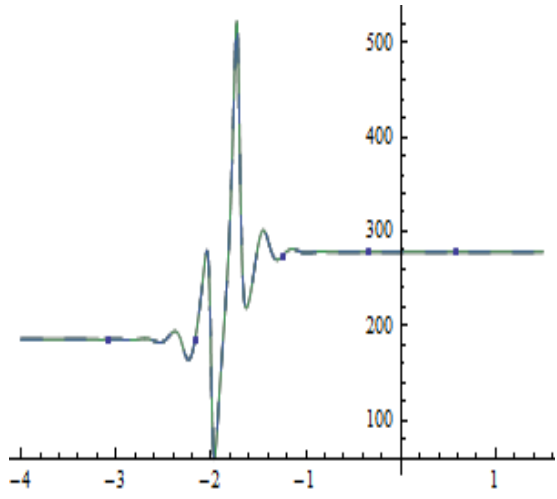

(c)

Figure 9. illustrated three-dimensions in (a); contour in (b) and two-dimensions in (c), Plots of $n_{40}(x, t)$ in Equation (37) for $a=1.3, b_{6}=7, b_{7}=-4, b_{8}=-5, \alpha=0.6$, respectively.

\subsection{Periodic Waves}

For periodic-wave interaction solutions, the bilinear form for $\Phi$ is as follows (See Figures 10 and 11):

$$
\Phi=\left(b_{2}+b_{1} \xi\right)^{2}+\left(b_{4}+b_{3} \xi\right)^{2}+\cos \left(b_{6}+b_{5} \xi\right)+b_{7}
$$

where $b_{i}(1 \leq i \leq 7)$ and all are real-valued parameters to be found. Inserting $\Phi$ into Equation (11) and collecting all powers of $\xi$ and $\cos \left(b_{5} \xi+b_{6}\right), \xi \cos \left(b_{5} \xi+b_{6}\right), \xi^{2} \cos \left(b_{5} \xi+b_{6}\right)$, $\xi^{3} \cos \left(b_{5} \xi+b_{6}\right), \quad \xi \sin \left(b_{5} \xi+b_{6}\right), \quad \xi^{2} \sin \left(b_{5} \xi+b_{6}\right), \quad \xi^{3} \sin \left(b_{5} \xi+b_{6}\right), \quad \xi \cos \left(b_{5} \xi+b_{6}\right)$ $\sin \left(b_{5} \xi+b_{6}\right), \xi^{2} \cos \left(b_{5} \xi+b_{6}\right) \sin \left(b_{5} \xi+b_{6}\right), \sin \left(b_{5} \xi+b_{6}\right)^{3}$, we obtain proper results as follows:

Set I. For $u_{0}=0$,

$$
\begin{gathered}
a=a, b=2 \sqrt{2} \sqrt{\frac{1}{a^{2}-1}}, \beta=-\frac{a^{4}-a^{2}+4 b_{5}^{2}}{2(a-1)(a+1)}, b_{1}=i b_{3}, b_{2}=b_{2}, b_{3}=b_{3}, b_{4}=b_{4}, b_{5}=b_{5}, \\
b_{6}=b_{6}, b_{7}=-b_{4}^{2}-b_{2}^{2} .
\end{gathered}
$$


By using these parameters in Equation (38), and then by using Equations (8) and (10), we obtain

$$
\begin{array}{r}
u(\xi)=\frac{2\left(2 i b_{3}\left(b_{2}+i b_{3} \xi\right)+2 b_{3}\left(b_{4}+b_{3} \xi\right)-b_{5} \sin \left(b_{6}+b_{5} \xi\right)\right)}{-b_{2}^{2}-b_{4}^{2}+\left(b_{2}+i b_{3} \xi\right)^{2}+\left(b_{4}+b_{3} \xi\right)^{2} \cos \left(b_{6}+b_{5} \xi\right)} \\
v(\xi)=\frac{8}{-1+a^{2}}\left(\frac{2 i b_{3}\left(b_{2}+i b_{3} \xi\right)+2 b_{3}\left(b_{4}+b_{3} \xi\right)-b_{5} \sin \left(b_{6}+b_{5} \xi\right)}{-b_{2}^{2}-b_{4}^{2}+\left(b_{2}+i b_{3} \xi\right)^{2}+\left(b_{4}+b_{3} \xi\right)^{2} \cos \left(b_{6}+b_{5} \xi\right)}\right)^{2} .
\end{array}
$$

Now, using Equation (5), we obtain the required solution for Equation (1):

$$
\begin{aligned}
& m_{1}(x, t)=\frac{2 e^{i\left(a x-\frac{\left(-a^{2}+a^{4}+4 b_{5}^{2}\right) t^{-s}(-1+\alpha)}{2\left(-1+a^{2}\right) B(\alpha) \sum_{s=0}^{\infty}\left(-\frac{\alpha}{1-\alpha}\right)^{s} \Gamma(1-\alpha)}\right)}\left(-b_{5} \sin \left(b_{6}+b_{5} \Omega\right)+2 i b_{3}\left(b_{2}+i b_{3} \Omega\right)+2 b_{3}\left(b_{4}+b_{3} \Omega\right)\right)}{-b_{2}^{2}-b_{4}^{2}+\cos \left(b_{6}+b_{5} \Omega\right)+\left(b_{2}+i b_{3} \Omega\right)^{2}+\left(b_{4}+b_{3} \Omega\right)^{2}}, \\
& n_{2}(x, t)=\frac{8}{\left(a^{2}-1\right)}\left(\frac{-b_{5} \sin \left(b_{6}+b_{5} \Omega\right)+2 i b_{3}\left(b_{2}+i b_{3} \Omega\right)+2 b_{3}\left(b_{4}+b_{3} \Omega\right)}{-b_{2}^{2}-b_{4}^{2}+\cos \left(b_{6}+b_{5} \Omega\right)+\left(b_{2}+i b_{3} \Omega\right)^{2}+\left(b_{4}+b_{3} \Omega\right)^{2}}\right)^{2},
\end{aligned}
$$

where $\Omega=2 \sqrt{2} \sqrt{\frac{1}{-1+a^{2}}} x-\frac{2 \sqrt{2} a \sqrt{\frac{1}{-1+a^{2}}} t^{-s}(-1+\alpha)}{B(\alpha) \sum_{s=0}^{\infty}\left(-\frac{\alpha}{1-\alpha}\right)^{5} \Gamma(1-\alpha s)}$.

Set II. For $b_{1}=b_{2}=0$,

$$
a=a, b=\frac{2 \sqrt{-\frac{2}{a^{2}-1} u_{0}}}{b_{5}}, \beta=-\frac{a^{4}-a^{2}+4 u_{0}^{2}}{2\left(a^{2}-1\right)}, b_{3}=b_{3}, b_{4}=b_{4}, b_{5}=b_{5}, b_{6}=b_{6}, b_{7}=b_{7}, u_{0}=u_{0} .
$$

By using these parameters in Equation (38), and then by using Equations (8) and (10) in Equation (5), we obtain

$$
\begin{aligned}
& m_{3}(x, t)=e^{i\left(a x-\frac{t^{-s}\left(-a^{2}+a^{4}+4 u_{0}^{2}\right)(-1+\alpha)}{2\left(-1+a^{2}\right) B(\alpha) \sum_{s=0}^{\infty}\left(-\frac{\alpha}{1-\alpha}\right)^{s} \Gamma(1-\alpha)}\right)}\left(u_{0}+\frac{2\left(-b_{5} \sin \left(b_{6}+b_{5} \Omega\right)+2 b_{3}\left(b_{4}+b_{3} \Omega\right)\right)}{b_{7}+\cos \left(b_{6}+b_{5} \Omega\right)+\left(b_{4}+b_{3} \Omega\right)^{2}}\right), \\
& n_{4}(x, t)=\frac{2}{-1+a^{2}}\left(u_{0}+\frac{2\left(-b_{5} \sin \left(b_{6}+b_{5} \Omega\right)+2 b_{3}\left(b_{4}+b_{3} \Omega\right)\right)}{b_{7}+\cos \left(b_{6}+b_{5} \Omega\right)+\left(b_{4}+b_{3} \Omega\right)^{2}}\right)^{2},
\end{aligned}
$$

$$
\text { where } \Omega=\frac{2 \sqrt{2} \sqrt{-\frac{u_{0}}{-1+a^{2}}} x}{b_{5}}-\frac{2 \sqrt{2} a t^{-s} \sqrt{-\frac{u_{0}}{-1+a^{2}}}(-1+\alpha)}{b_{5} B(\alpha) \sum_{s=0}^{\infty}\left(-\frac{\alpha}{1-\alpha}\right)^{s} \Gamma(1-\alpha s)} \text {. }
$$

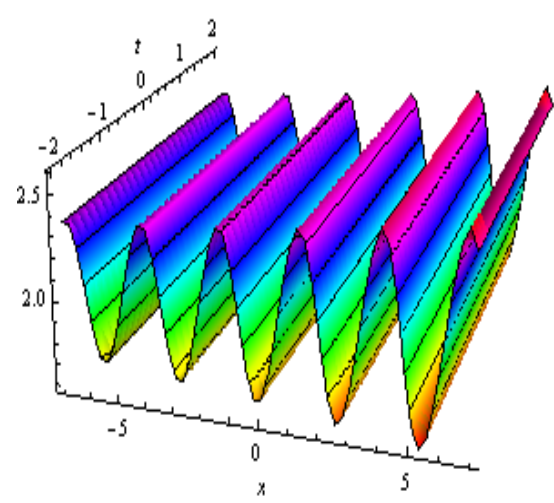

(a)

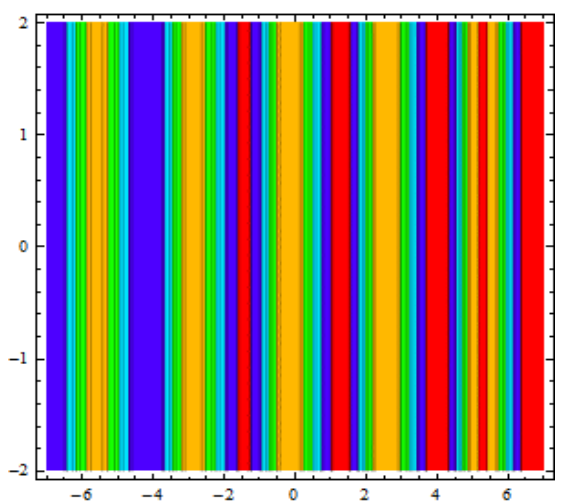

(b)

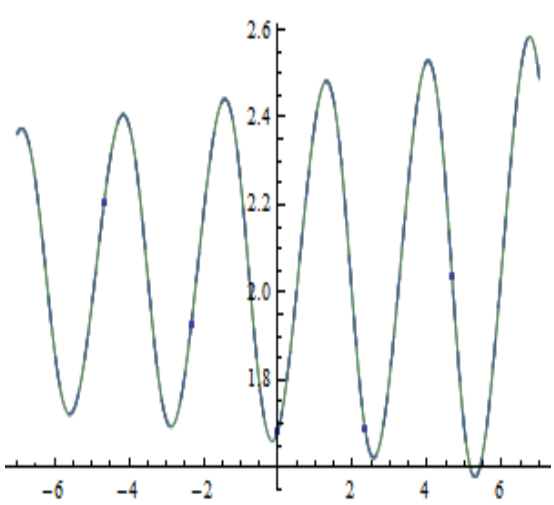

(c)

Figure 10. showed three-dimensions in (a); contour in (b) and two-dimensions in (c), Plots of $m_{3}(x, t)$ in Equation (43) at $a=2, u_{0}=-2, b_{3}=0.05, b_{4}=-3, b_{5}=2, b_{6}=5$, $b_{7}=1, \alpha=0.9$, respectively. 


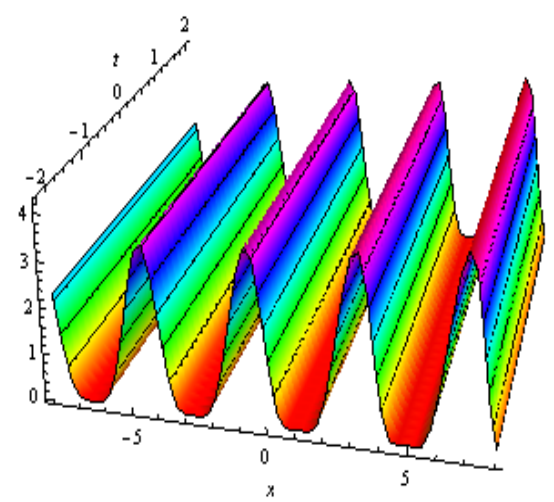

(a)

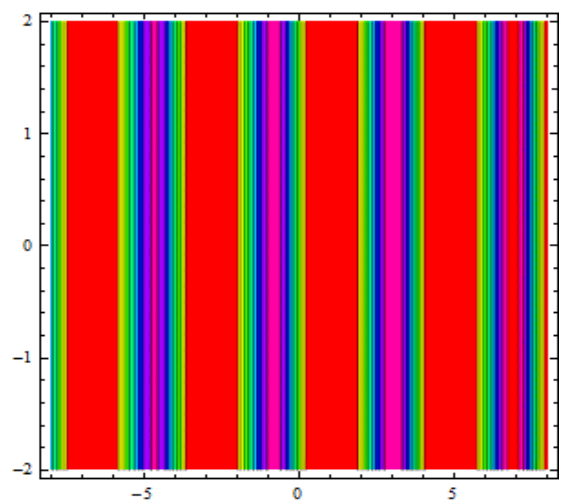

(b)

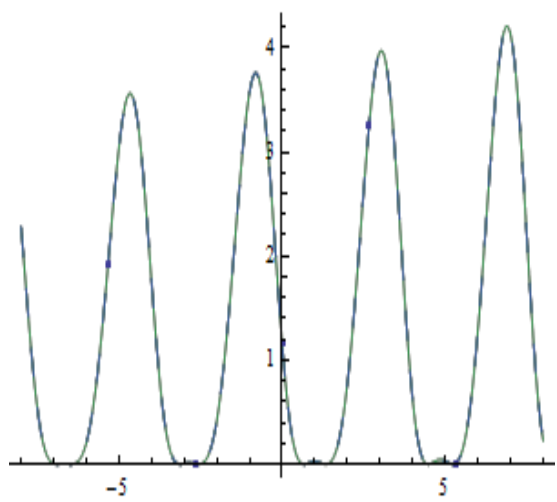

(c)

Figure 11. represented three-dimensions in (a); contour in (b) and two-dimensions in (c), Plots of $n_{4}(x, t)$ in Equation (43) at $a=2, u_{0}=-1, b_{3}=0.1, b_{4}=-3, b_{5}=7, b_{6}=3$, $b_{7}=2, \alpha=0.5$, respectively.

\section{Multiwave Solutions}

For multiwave solutions, $\Phi$ in bilinear form can be assumed as [32]

$$
\Phi=z_{0} \cosh \left(b_{2}+b_{1} \xi\right)+z_{1} \cos \left(b_{4}+b_{3} \xi\right)+z_{2} \cosh \left(b_{6}+b_{5} \xi\right),
$$

where $z_{i}^{\prime} s$ and $b_{i}^{\prime} s$ all are real-valued parameters to be measured. Inserting $\Phi$ into Equation (11) and collecting all coefficients of $\cosh \left(b_{2}+b_{1} \xi\right), \sinh \left(b_{2}+b_{1} \xi\right), \cos \left(b_{4}+b_{3} \xi\right) \sinh \left(b_{2}+b_{1} \xi\right)$ $\sinh \left(b_{6}+b_{5} \xi\right), \cos \left(b_{4}+b_{3} \xi\right), \cosh \left(b_{6}+b_{5} \xi\right), \sin \left(b_{4}+b_{3} \xi\right), \cosh \left(b_{2}+b_{1} \xi\right) \sinh \left(b_{6}+b_{5} \xi\right)$, $\cosh \left(b_{2}+b_{1} \xi\right) \cos \left(b_{4}+b_{3} \xi\right) \cosh \left(b_{6}+b_{5} \xi\right)$, and $\sinh \left(b_{2}+b_{1} \xi\right) \sin \left(b_{4}+b_{3} \xi\right) \sinh \left(b_{6}+b_{5} \xi\right)$, we obtain proper results, as follows (See Figures 12 and 13):

Case I.

$$
\begin{gathered}
a=a, b=2 \sqrt{2} \sqrt{\frac{1}{a^{2}-1}}, \beta=-\frac{4 u_{0}^{2}-a^{2}+a^{4}}{2\left(a^{2}-1\right)}, b_{1}=\frac{i \sqrt{2} u_{0}}{2}, b_{2}=b_{2}, b_{3}=\frac{\sqrt{2} u_{0}}{2}, b_{4}=b_{4}, \\
b_{5}=\frac{i \sqrt{2} u_{0}}{2}, b_{6}=b_{6}, u_{0}=u_{0}, z_{0}=z_{0}, z_{1}=z_{1}, z_{2}=z_{2} .
\end{gathered}
$$

By using these values in Equation (44) and then by using Equations (8) and (10), we obtain

$$
\begin{aligned}
& u(\xi)=u_{0}-\frac{\sqrt{2} u_{0}\left(z_{1} \sin \left(b_{4}+\frac{u_{0} \tilde{\xi}}{\sqrt{2}}\right)-i z_{0} \sinh \left(b_{2}+\frac{i u_{0} \tilde{\xi}}{\sqrt{2}}\right)-i z_{2} \sinh \left(b_{6}+\frac{i u_{0} \tilde{\xi}}{\sqrt{2}}\right)\right)}{z_{1} \cos \left(b_{4}+\frac{u_{0} \zeta}{\sqrt{2}}\right)+z_{0} \cosh \left(b_{2}+\frac{i u_{0} \xi}{\sqrt{2}}\right)+z_{2} \cosh \left(b_{6}+\frac{i u_{0} \zeta}{\sqrt{2}}\right)}, \\
& v(\xi)=\frac{2}{a^{2}-1}\left(u_{0}-\frac{\sqrt{2} u_{0}\left(z_{1} \sin \left(b_{4}+\frac{u_{0} \xi}{\sqrt{2}}\right)-i z_{0} \sinh \left(b_{2}+\frac{i u_{0} \xi}{\sqrt{2}}\right)-i z_{2} \sinh \left(b_{6}+\frac{i u_{0} \xi}{\sqrt{2}}\right)\right)}{z_{1} \cos \left(b_{4}+\frac{u_{0} \xi}{\sqrt{2}}\right)+z_{0} \cosh \left(b_{2}+\frac{i u_{0} \xi}{\sqrt{2}}\right)+z_{2} \cosh \left(b_{6}+\frac{i u_{0} \xi}{\sqrt{2}}\right)}\right)^{2} .
\end{aligned}
$$

Using Equation (5), we obtain the following multiwave solutions for Equation (1):

$$
m_{41}(x, t)=e^{i\left(a x+\frac{t^{-s}\left(4 u_{0}^{2}-a^{2}+a^{4}\right)(-1+\alpha)}{2\left(a^{2}-1\right) B(\alpha) \sum_{s=0}^{\infty}\left(-\frac{\alpha}{1-\alpha}\right)^{S} \Gamma(1-\alpha)}\right)}\left(u_{0}-\frac{\sqrt{2} u_{0}\left(z_{1} \sin \left(b_{4}+\Lambda\right)-i z_{0} \sinh \left(b_{2}+i \Lambda\right)-i z_{2} \sinh \left(b_{6}+i \Lambda\right)\right)}{z_{1} \cos \left(b_{4}+\Lambda\right)+z_{0} \cosh \left(b_{2}+i \Lambda\right)+z_{2} \cosh \left(b_{6}+i \Lambda\right)}\right),
$$

$n_{42}(x, t)=\frac{2}{\left(a^{2}-1\right)}\left(u_{0}-\frac{\sqrt{2} u_{0}\left(z_{1} \sin \left(b_{4}+\Lambda\right)-i z_{0} \sinh \left(b_{2}+i \Lambda\right)-i z_{2} \sinh \left(b_{6}+i \Lambda\right)\right)}{z_{1} \cos \left(b_{4}+\Lambda\right)+z_{0} \cosh \left(b_{2}+i \Lambda\right)+z_{2} \cosh \left(b_{6}+i \Lambda\right)}\right)^{2}$

where $\Lambda=2 \sqrt{\frac{1}{a^{2}-1}} u_{0}\left(x+\frac{a t^{-s}(-1+\alpha)}{B(\alpha) \sum_{s=0}^{\infty}\left(-\frac{\alpha}{1-\alpha}\right)^{s} \Gamma(1-\alpha s)}\right)$ 


\section{Case II.}

$$
\begin{aligned}
a=a, b=\frac{2 \sqrt{-\frac{1}{a^{2}-1}} u_{0}}{b_{5}}, \beta & =-\frac{4 u_{0}^{2}+a^{4}-a^{2}}{2(a-1)(a+1)}, b_{1}=-b_{5}, b_{2}=b_{2}, b_{3}=i b_{5}, b_{4}=b_{4}, b_{5}=b_{5}, \\
b_{6} & =b_{6}, u_{0}=u_{0}, z_{0}=z_{0}, z_{1}=z_{1}, z_{2}=z_{2} .
\end{aligned}
$$

By using these values in Equation (44) and then by using Equations (8) and (10), we obtain

$$
\begin{gathered}
u(\xi)=u_{0}+\frac{2 b_{5}\left(-i z_{1} \sin \left(b_{4}+i b_{5} \xi\right)-z_{0} \sinh \left(b_{2}-b_{5} \xi\right)+z_{2} \sinh \left(b_{6}+b_{5} \xi\right)\right)}{z_{1} \cos \left(b_{4}+i b_{5} \xi\right)+z_{0} \cosh \left(b_{2}-b_{5} \xi\right)+z_{2} \cosh \left(b_{6}+b_{5} \xi\right)}, \\
v(\xi)=\frac{2}{-1+a^{2}}\left(u_{0}+\frac{2 b_{5}\left(-i z_{1} \sin \left(b_{4}+i b_{5} \xi\right)-z_{0} \sinh \left(b_{2}-b_{5} \xi\right)+z_{2} \sinh \left(b_{6}+b_{5} \xi\right)\right)}{z_{1} \cos \left(b_{4}+i b_{5} \xi\right)+z_{0} \cosh \left(b_{2}-b_{5} \xi\right)+z_{2} \cosh \left(b_{6}+b_{5} \xi\right)}\right)^{2} .
\end{gathered}
$$

Using Equation (5), we obtain the following multiwave solutions for Equation (1):

$$
m_{43}(x, t)=e^{i\left(a x+\frac{t^{-s}\left(4 u_{0}^{2}-a^{2}+a^{4}\right)(-1+\alpha)}{2\left(a^{2}-1\right) B(\alpha) \sum_{s=0}^{\infty}\left(-\frac{\alpha}{1-\alpha}\right)^{S} \Gamma(1-\alpha)}\right)}\left(u_{0}+\frac{2 b_{5}\left(-i z_{1} \sin \left(b_{4}+i \Lambda\right)-z_{0} \sinh \left(b_{2}-\Lambda\right)+z_{2} \sinh \left(b_{6}+\Lambda\right)\right)}{z_{1} \cos \left(b_{4}+i \Lambda\right)+z_{0} \cosh \left(b_{2}-\Lambda\right)+z_{2} \cosh \left(b_{6}+\Lambda\right)},\right.
$$$$
n_{44}(x, t)=\frac{2}{\left(a^{2}-1\right)}\left(u_{0}+\frac{2 b_{5}\left(-i z_{1} \sin \left(b_{4}+i \Lambda\right)-z_{0} \sinh \left(b_{2}-\Lambda\right)+z_{2} \sinh \left(b_{6}+\Lambda\right)\right)}{z_{1} \cos \left(b_{4}+i \Lambda\right)+z_{0} \cosh \left(b_{2}-\Lambda\right)+z_{2} \cosh \left(b_{6}+\Lambda\right)}\right)^{2},
$$

$$
\text { where } \Lambda=2 \sqrt{\frac{1}{1-a^{2}}} u_{0}\left(x+\frac{a t^{-s}(-1+\alpha)}{B(\alpha) \sum_{s=0}^{\infty}\left(-\frac{\alpha}{1-\alpha}\right)^{s} \Gamma(1-\alpha)}\right) \text {. }
$$

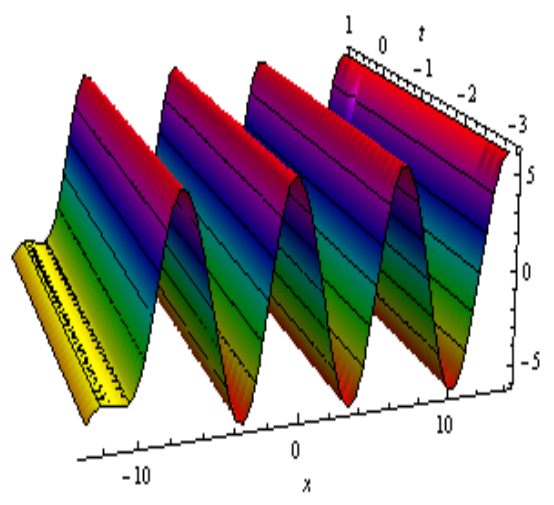

(a)

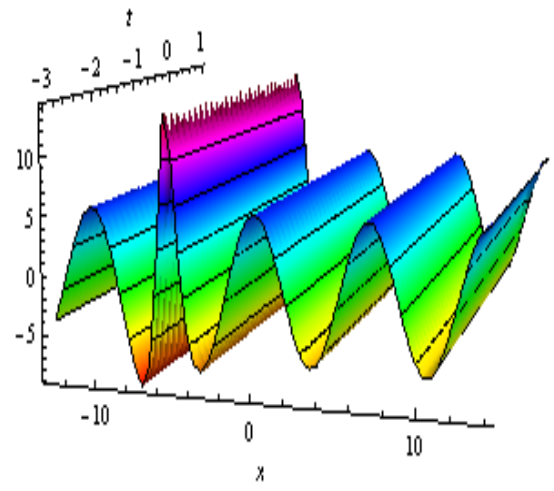

(d)

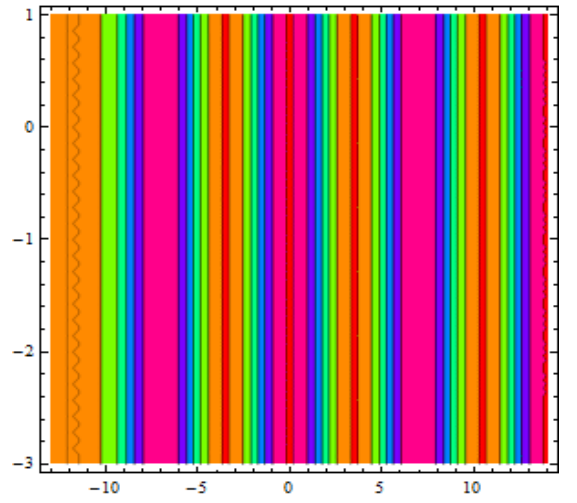

(b)

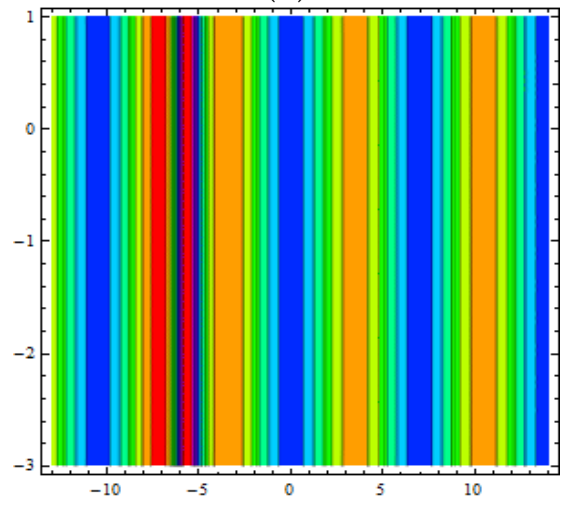

(e)

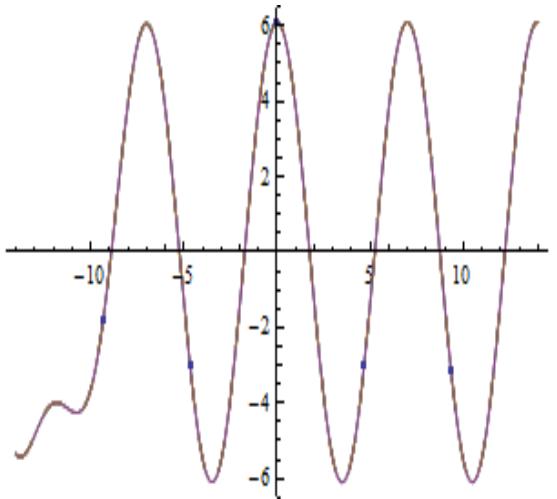

(c)

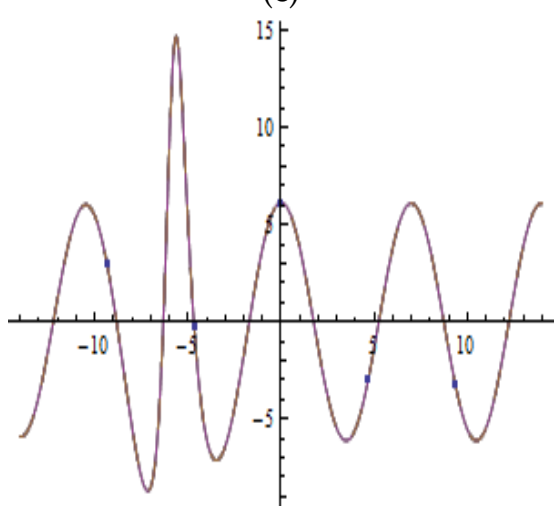

(f)

Figure 12. Showed three-dimensions in (a); contour in (b) and two-dimensions in (c), Graphical representation of $m_{43}(x, t)$ in Equation (50), for $a=0.9, u_{0}=0.1, b_{2}=-5$, $b_{4}=5, b_{5}=3, z_{0}=-2, z_{1}=1, z_{2}=2, \alpha=0.9$, respectively. 


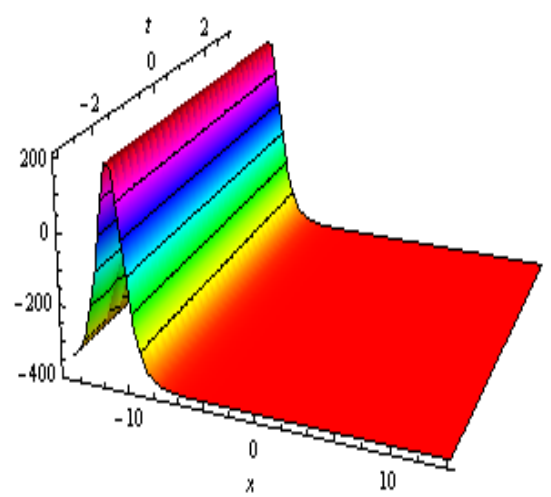

(a)

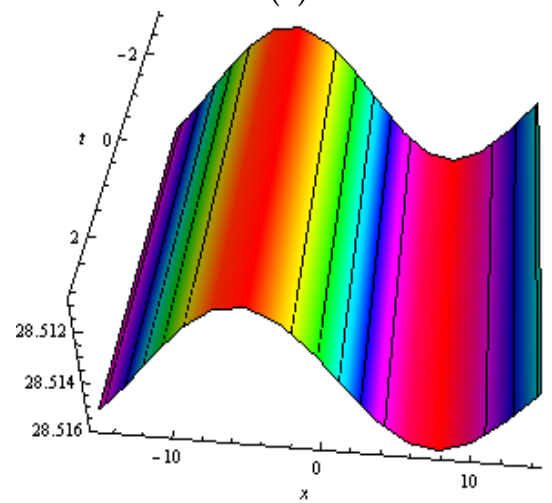

(d)

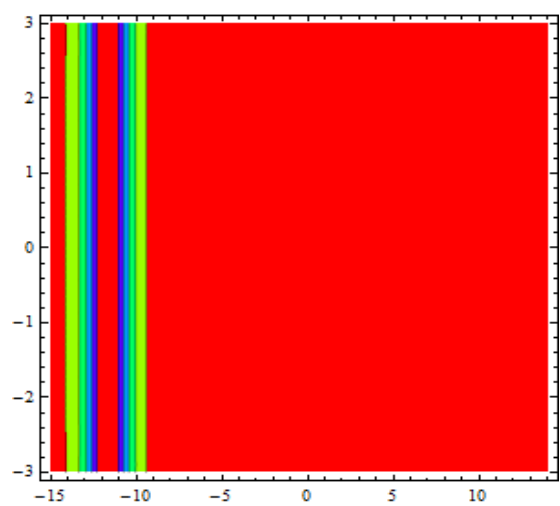

(b)

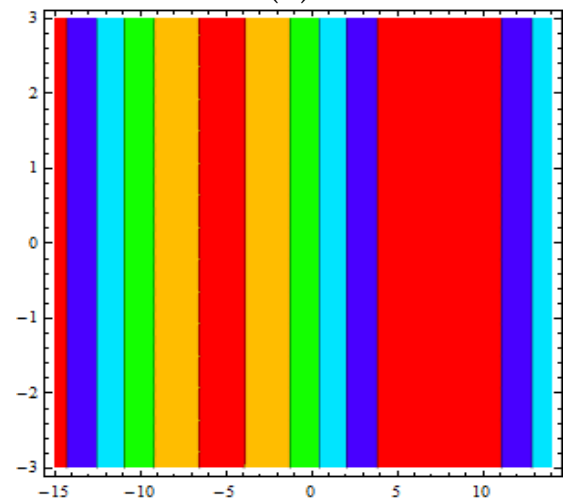

(e)

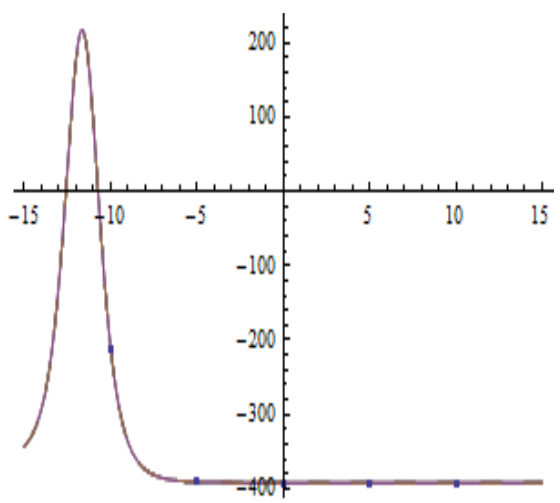

(c)

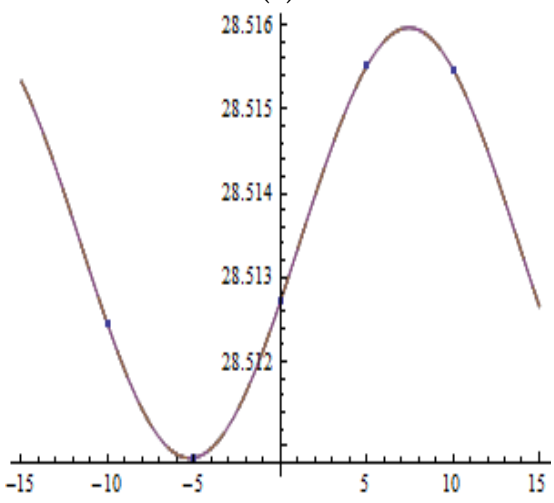

(f)

Figure 13. Represented three-dimensions in (a); contour in (b) and two-dimensions in (c), Graphical representation of $n_{44}(x, t)$ in Equation (50), for $u_{0}=0.1, b_{2}=-5, b_{4}=5$, $b_{5}=3, b_{6}=10, z_{0}=-2, z_{1}=1, z_{2}=2, \alpha=0.9$, respectively.

\section{Homoclinic Breather Approach}

To obtain breather solutions, $\Phi$ in bilinear form can be assumed as [32]

$$
\Phi=e^{-p\left(b_{1} \xi+b_{2}\right)}+z_{1} e^{p\left(b_{3} \xi+b_{4}\right)}+z_{2} \cos \left(q\left(b_{5} \xi+b_{6}\right)\right)
$$

where $p, q, z_{1}, z_{2}$, and $b_{i}^{\prime} s$ all are real-valued parameters to be found. Inserting $\Phi$ into Equation (11) and collecting all coefficients of $e^{p\left(b_{4}+b_{3} \xi\right)}, \sin \left(q\left(b_{6}+b_{5} \xi\right)\right), \cos \left(q\left(b_{6}+b_{5} \xi\right)\right)$, $e^{p\left(b_{4}+b_{3} \xi\right)} \sin \left(q\left(b_{6}+b_{5} \xi\right)\right), e^{-p\left(b_{2}+b_{1} \xi\right)+p\left(b_{4}+b_{3} \xi\right)} \cos \left(q\left(b_{6}+b_{5} \xi\right)\right)$, and $\cos \left(q\left(b_{6}+b_{5} \xi\right)\right)$ $\sin \left(q\left(b_{6}+b_{5} \xi\right)\right)$, we obtain an algebraic system of equations, then, after solving them, we obtain proper results, as follows (See Figures 14 and 15):

Case I.

$$
\begin{gathered}
a=a, b=2 \sqrt{2} \sqrt{\frac{1}{a^{2}-1}}, \beta=-\frac{a^{4}-16 b_{5}^{2} q^{2}-a^{2}}{2\left(a^{2}-1\right)}, b_{1}=\frac{i q b_{5}}{p}, b_{2}=b_{2}, b_{3}=\frac{i q b_{5}}{p}, b_{4}=b_{4}, b_{5}=b_{5} \\
b_{6}=b_{6}, u_{0}=0, p=p, q=q, z_{1}=z_{1}, z_{2}=z_{2} .
\end{gathered}
$$

By using these parameters in Equation (51) and then by using Equations (8) and (10), we obtain

$$
\begin{gathered}
u(\xi)=\frac{2 i b_{5} q\left(-1+e^{b_{2} p+b_{4} p+2 i b_{5} q \xi} z_{1}+i e^{b_{2} p+i b_{5} q \xi_{\xi}} z_{2} \sin \left(q\left(b_{6}+b_{5} \xi\right)\right)\right)}{1+e^{b_{2} p+b_{4} p+2 i b_{5} q \xi} z_{1}+e^{b_{2} p+i b_{5} q \xi^{\tau}} z_{2} \cos \left(q\left(b_{6}+b_{5} \xi\right)\right)} \\
v(\xi)=-\frac{8 b_{5}^{2} q^{2}}{a^{2}-1}\left(\frac{-1+e^{b_{2} p+b_{4} p+2 i b_{5} q \xi} z_{1}+i e^{b_{2} p+i b_{5} q \xi} z_{2} \sin \left(q\left(b_{6}+b_{5} \xi\right)\right)}{1+e^{b_{2} p+b_{4} p+2 i b_{5} q \xi} z_{1}+e^{b_{2} p+i b_{5} q q_{5}} z_{2} \cos \left(q\left(b_{6}+b_{5} \xi\right)\right)}\right)^{2} .
\end{gathered}
$$

Using Equation (5), we obtain the following breather solutions for Equation (1): 


$$
\begin{gathered}
m_{51}(x, t)=\frac{2 i b_{5} e^{\Delta} q\left(-1+e^{b_{2} p+b_{4} p+4 i \Omega} z_{1}+i e^{b_{2} p+2 i \Omega} z_{2} \sin \left(q\left(b_{6}+\frac{2}{q} \Omega\right)\right)\right)}{1+e^{b_{2} p+b_{4} p+4 i \Omega_{1}} z_{1}+e^{b_{2} p+2 i \Omega} z_{2} \cos \left(q\left(b_{6}+\frac{2}{q} \Omega\right)\right)}, \\
n_{52}(x, t)=-\frac{8 b_{5}^{2} q^{2}}{\left(-1+a^{2}\right)}\left(\frac{-1+e^{b_{2} p+b_{4} p+4 i \Omega} z_{1}+i e^{b_{2} p+2 i \Omega} z_{2} \sin \left(q\left(b_{6}+\frac{2}{q} \Omega\right)\right)}{1+e^{b_{2} p+b_{4} p+4 i \Omega^{2}} z_{1}+e^{b_{2} p+2 i \Omega} z_{2} \cos \left(q\left(b_{6}+\frac{2}{q} \Omega\right)\right)}\right)^{2}, \\
\text { where } \Delta=\frac{1}{2} i\left(2 a x+\frac{\left(-16 b_{5}^{2} q^{2}-a^{2}+a^{4}\right) t^{-s}(\alpha-1)}{(-1+a)(1+a) B(\alpha) \sum_{s=0}^{\infty}\left(-\frac{\alpha}{1-\alpha}\right)^{s} \Gamma(1-\alpha s)}\right), \\
\Omega=\sqrt{2} \sqrt{\frac{1}{-1+a^{2}}} b_{5} q\left(x+\frac{a t^{-s}(-1+\alpha)}{B(\alpha) \sum_{s=0}^{\infty}\left(-\frac{\alpha}{1-\alpha}\right)^{s} \Gamma(1-\alpha s)}\right) .
\end{gathered}
$$

\section{Case II.}

$$
\begin{gathered}
a=a, b=2 \sqrt{2} \sqrt{\frac{1}{a^{2}-1}}, \beta=-\frac{a^{4}+4 u_{0}^{2}-a^{2}}{2\left(a^{2}-1\right)}, b_{1}=\frac{u_{0}}{p}, b_{2}=b_{2}, b_{3}=-\frac{u_{0}}{p}, b_{4}=b_{4}, b_{5}=0 \\
b_{6}=b_{6}, u_{0}=u_{0}, p=p, q=q, z_{1}=z_{1}, z_{2}=z_{2} .
\end{gathered}
$$

By using these parameters in Equation (51), and then by using Equations (8) and (10) in Equation (5), we obtain the following solutions for Equation (1):

$$
m_{53}(x, t)=-\frac{e^{\frac{1}{2} i\left(2 a x+\frac{t^{-s}\left(4 u_{0}^{2}-a^{2}+a^{4}\right)(\alpha-1)}{(-1+a)(1+a) B(\alpha) \sum_{s=0}^{\infty}\left(-\frac{\alpha}{1-\alpha}\right)^{s} \Gamma(1-\alpha s)}\right)} u_{0}\left(1+e^{b_{2} p+b_{4} p} z_{1}-e^{p(\Omega)} z_{2} \cos \left(b_{6} q\right)\right)}{1+e^{b_{2} p+b_{4} p} z_{1}+e^{p(\Omega)} z_{2} \cos \left(b_{6} q\right)},
$$

$$
n_{54}(x, t)=\frac{2 u_{0}^{2}}{\left(-1+a^{2}\right)}\left(\frac{1+e^{b_{2} p+b_{4} p} z_{1}-e^{p(\Omega)} z_{2} \cos \left(b_{6} q\right)}{1+e^{b_{2} p+b_{4} p_{2}} z_{1}+e^{p(\Omega)} z_{2} \cos \left(b_{6} q\right)}\right)^{2}
$$

$$
\text { where } \Omega=b_{2}+\frac{2 \sqrt{2} \sqrt{\frac{1}{-1+a^{2}}} u_{0}\left(x+\frac{a t^{-s}(-1+\alpha)}{B(\alpha) \sum_{s=0}^{\infty}\left(-\frac{\alpha}{1-\alpha}\right)^{S} \Gamma(1-\alpha s)}\right)}{p} \text {. }
$$

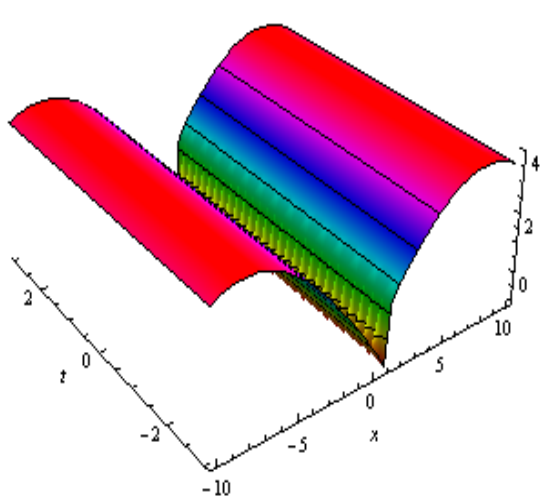

(a)

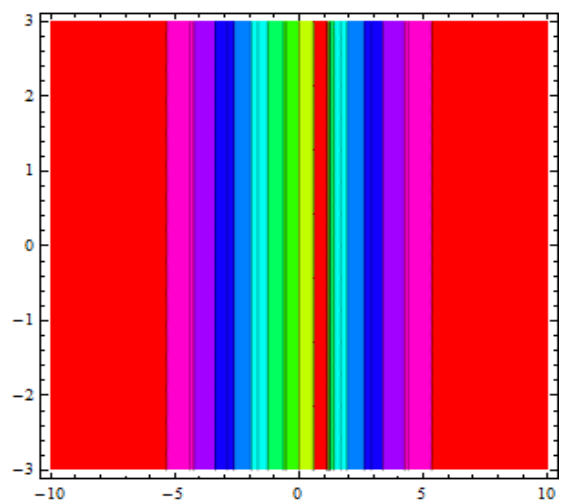

(b)

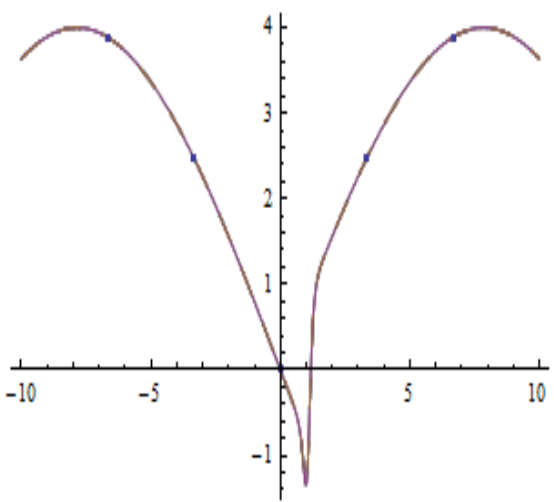

(c)

Figure 14. Cont. 


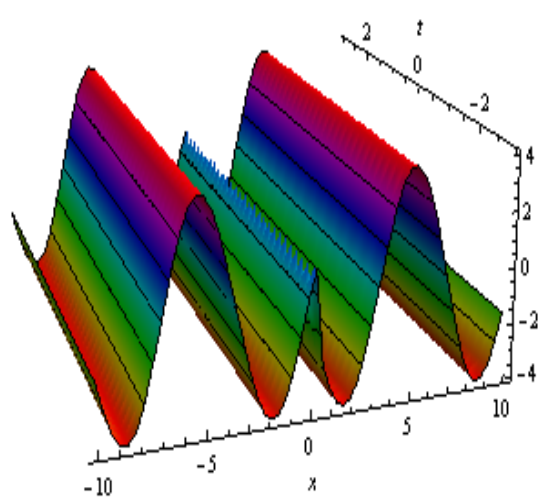

(d)

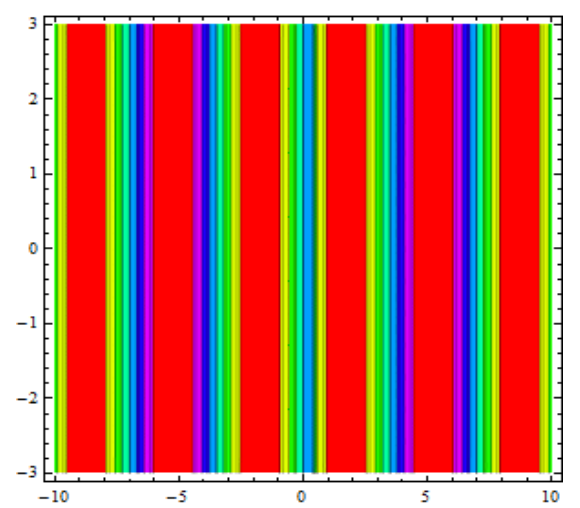

(e)

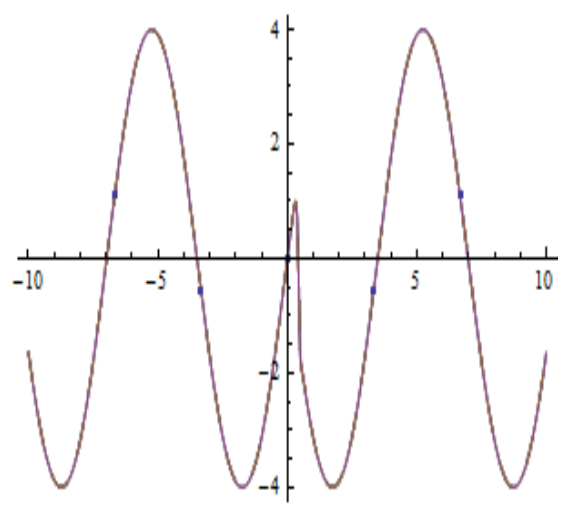

(f)

Figure 14. Explain three-dimensions in (a); contour in (b) and two-dimensions in (c), Graphical representation of $m_{51}(x, t)$ in Equation (54), at $b_{2}=-4, b_{4}=-7, b_{5}=10$, $b_{6}=3, z_{1}=-2, z_{2}=2, p=3, q=-0.2, \alpha=0.8$, respectively.

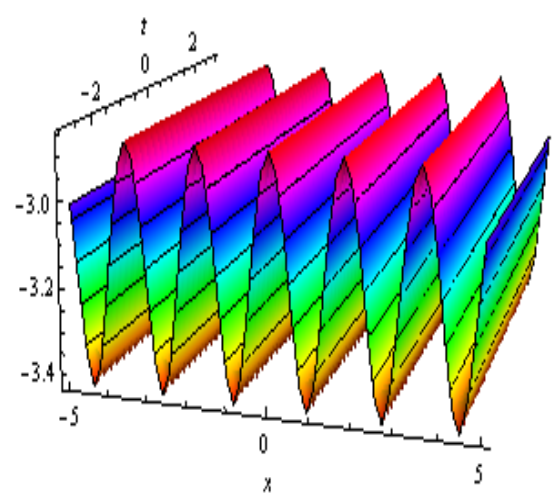

(a)

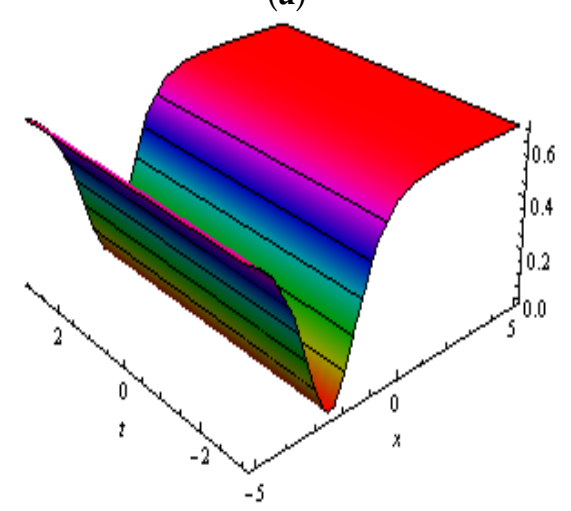

(d)

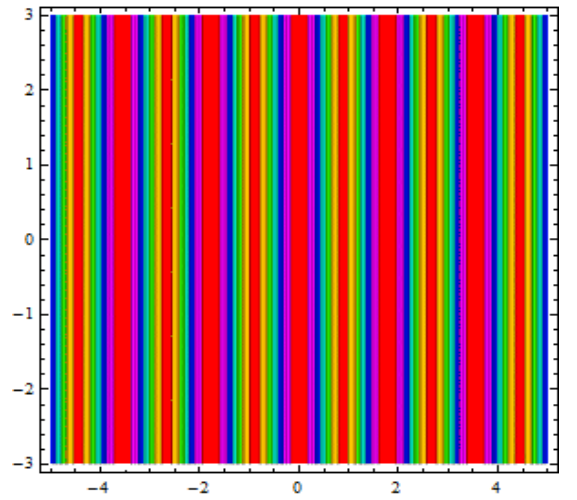

(b)

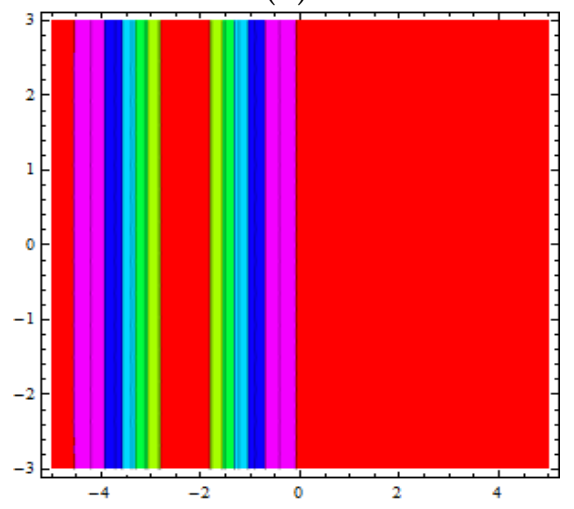

(e)

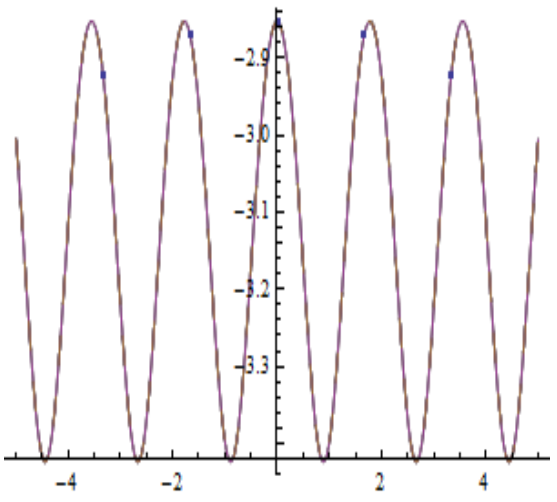

(c)

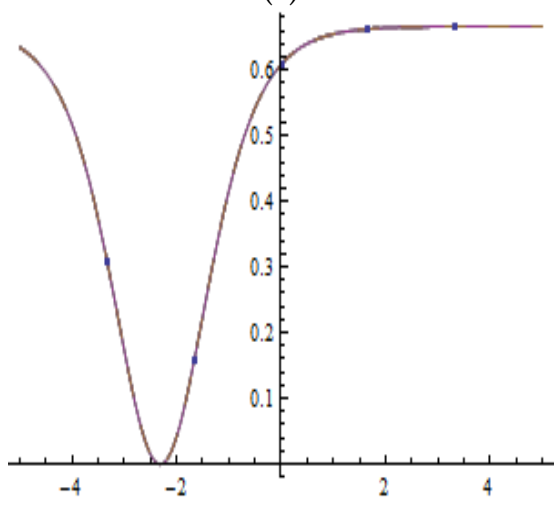

(f)

Figure 15. Clarify three-dimensions in (a); contour in (b) and two-dimensions in (c), Graphical representation of $n_{54}(x, t)$ in Equation (56), at $b_{2}=4, b_{4}=-3, b_{6}=3$, $z_{1}=-1.5, z_{2}=2.5, p=1, q=-1, u_{0}=1, \alpha=0.6$, successively.

\section{The Periodic Cross-Kink Wave Solutions}

For this, $\Phi$ in bilinear form can be assumed as [33]

$$
\Phi=e^{-\left(b_{1} \xi+b_{2}\right)}+z_{1} e^{b_{1} \xi+b_{2}}+z_{2} \cos \left(b_{3} \xi+b_{4}\right)+z_{3} \cosh \left(b_{5} \xi+b_{6}\right)+b_{7},
$$

where $z_{i}^{\prime} s$ and $b_{i}^{\prime} s$ all are real-valued parameters to be measured. Inserting $\Phi$ into Equation (11) and collecting all coefficients of $e^{b_{1} \xi+b_{2}}, e^{-b_{1} \xi-b_{2}}, e^{b_{2}+b_{1} \xi+2\left(b_{2}+b_{1} \xi\right)}, e^{-\left(b_{1} \xi+b_{2}\right)+2\left(b_{1} \xi+b_{2}\right)}$, $\cos \left(b_{4}+b_{3} \xi\right), \quad \cos \left(b_{4}+b_{3} \xi\right) \cosh \left(b_{6}+b_{5} \xi\right), \quad e^{-\left(b_{1} \xi+b_{2}\right)+2\left(b_{1} \xi+b_{2}\right)} \cos \left(b_{4}+b_{3} \xi\right)$, 
$e^{-\left(b_{1} \xi+b_{2}\right)+2\left(b_{1} \xi+b_{2}\right)} \sin \left(b_{4}+b_{3} \xi\right), \quad \cos \left(b_{4}+b_{3} \xi\right) \cosh \left(b_{6}+b_{5} \xi\right) \sinh \left(b_{6}+b_{5} \xi\right)$, $e^{-\left(b_{1} \xi+b_{2}\right)+2\left(b_{1} \xi+b_{2}\right)} \cos \left(b_{4}+b_{3} \xi\right) \cosh \left(b_{6}+b_{5} \xi\right)$, and $e^{\left.-\left(b_{1} \xi+b_{2}\right)+2 b_{1} \xi+2 b_{2}\right)} \sin \left(b_{4}+b_{3} \xi\right)$ $\sinh \left(b_{6}+b_{5} \xi\right)$, after solving them, we attain the following parameters (See Figures 16 and 17):

Case I. For $b_{4}=0$,

$$
\begin{gathered}
a=a, b=8 \sqrt{2} \sqrt{\frac{1}{a^{2}-1}}, \beta=-\frac{64 b_{1}^{2}-a^{2}+a^{4}}{2\left(a^{2}-1\right)}, b_{1}=b_{1}, b_{2}=b_{2}, b_{3}=b_{3}, b_{5}=0, b_{6}=b_{6}, \\
b_{7}=0, u_{0}=-2 b_{1}, z_{1}=z_{1}, z_{2}=0, z_{3}=z_{3} .
\end{gathered}
$$

By using these values in Equation (57), and then by using Equations (8) and (10), we obtain

$$
\begin{gathered}
u(\xi)=-2 b_{1}+\frac{2\left(-b_{1} e^{-b_{2}-b_{1} \tilde{\xi}}+b_{1} e^{b_{2}+b_{1}} \tilde{\xi}_{z_{1}}\right)}{e^{-b_{2}-b_{1} \tilde{\xi}}+e^{b_{2}+b_{1} \tilde{\xi}_{1}+z_{3} \cosh \left(b_{6}\right)}} \\
v(\xi)=\frac{2}{a^{2}-1}\left(-2 b_{1}+\frac{2\left(-b_{1} e^{-b_{2}-b_{1}} \tilde{\xi}+b_{1} e^{b_{2}+b_{1}} \tilde{\xi}_{z_{1}}\right)}{e^{-b_{2}-b_{1} \tilde{\xi}}+e^{b_{2}+b_{1}} z_{1}+z_{3} \cosh \left(b_{6}\right)}\right)^{2} .
\end{gathered}
$$

Now, using Equation (5), we obtain the following solutions for Equation (1):

$$
\begin{aligned}
& m_{61}(x, t)=-\frac{2 b_{1} e^{\frac{i}{2}\left(2 a x+\frac{\left(64 b_{1}^{2}-a^{2}+a^{4}\right) t^{-s}(\alpha-1)}{\left(a^{2}-1\right) B(\alpha) \sum_{s=0}^{\infty}\left(-\frac{\alpha}{1-\alpha}\right)^{S} \Gamma(1-\alpha s)}\right)}\left(2+e^{\Omega} z_{3} \cosh \left(b_{6}\right)\right)}{1+e^{2 \Omega} z_{1}+e^{\Omega} z_{3} \cosh \left(b_{6}\right)}, \\
& n_{62}(x, t)=\frac{8 b_{1}^{2}}{\left(a^{2}-1\right)}\left(\frac{2+e^{\Omega} z_{3} \cosh \left(b_{6}\right)}{1+e^{2 \Omega} z_{1}+e^{\Omega} z_{3} \cosh \left(b_{6}\right)}\right)^{2},
\end{aligned}
$$

where $\Omega=b_{2}+8 \sqrt{2} \sqrt{\frac{1}{a^{2}-1}} b_{1}\left(x+\frac{a t^{-s}(-1+\alpha)}{B(\alpha) \sum_{s=0}^{\infty}\left(-\frac{\alpha}{1-\alpha}\right)^{s} \Gamma(1-\alpha s)}\right)$.

\section{Case II.}

$$
\begin{gathered}
a=a, b=2 \sqrt{2} \sqrt{\frac{1}{a^{2}-1}}, \beta=-\frac{a^{4}-a^{2}-16 b_{3}^{2}}{2\left(a^{2}-1\right)}, b_{1}=i b_{3}, b_{2}=b_{2}, b_{3}=b_{3}, b_{5}=-i b_{3}, b_{6}=b_{6}, \\
b_{7}=0, z_{1}=z_{1}, z_{2}=z_{2}, z_{3}=z_{3} .
\end{gathered}
$$

Now, by using these values in Equation (57), and then by using Equations (8) and (10) in Equation (5), we obtain the following solutions for Equation (1):

$$
\begin{gathered}
m_{63}(x, t)=\frac{2 i b_{3} e^{\Lambda}\left(-2+2 e^{2 b_{2}+\Omega} z_{1}-e^{b_{2}} z_{2}+e^{b_{2}+\Omega} z_{2}+e^{b_{2}}\left(-1+e^{\Omega}\right) z_{3} \cosh \left(b_{6}\right)-e^{b_{2}}\left(1+e^{\Omega}\right) z_{3} \sinh \left(b_{6}\right)\right)}{2+2 e^{2 b_{2}+\Omega} z_{1}+e^{b_{2}} z_{2}+e^{b_{2}+\Omega} z_{2}+e^{b_{2}}\left(1+e^{\Omega}\right) z_{3} \cosh \left(b_{6}\right)-e^{b_{2}}\left(-1+e^{\Omega}\right) z_{3} \sinh \left(b_{6}\right)}, \\
n_{64}(x, t)=-\frac{8 b_{3}^{2}}{\left(a^{2}-1\right)}\left(\frac{2-2 e^{2 b_{2}+\Omega} z_{1}+e^{b_{2}} z_{2}-e^{b_{2}+\Omega} z_{2}-e^{b_{2}}\left(-1+e^{\Omega}\right) z_{3} \cosh \left(b_{6}\right)+e^{b_{2}}\left(1+e^{\Omega}\right) z_{3} \sinh \left(b_{6}\right)}{2+2 e^{2 b_{2}+\Omega} z_{1}+e^{b_{2}} z_{2}+e^{b_{2}+\Omega} z_{2}+e^{b_{2}}\left(1+e^{\Omega}\right) z_{3} \cosh \left(b_{6}\right)-e^{b_{2}}\left(-1+e^{\Omega}\right) z_{3} \sinh \left(b_{6}\right)}\right)^{2}, \\
\text { where } \Lambda=\frac{1}{2} i\left(2 a x+\frac{\left(-16 b_{3}^{2}-a^{2}+a^{4}\right) t^{-s}(\alpha-1)}{\left(a^{2}-1\right) B(\alpha) \sum_{s=0}^{\infty}\left(-\frac{\alpha}{1-\alpha}\right)^{s} \Gamma(1-\alpha s)}\right), \\
\text { and } \Omega=4 i \sqrt{2} \sqrt{\frac{1}{-1+a^{2}}} b_{3}\left(x+\frac{a t^{-s}(-1+\alpha)}{B(\alpha) \sum_{s=0}^{\infty}\left(-\frac{\alpha}{1-\alpha}\right) s^{s} \Gamma(1-\alpha s)}\right) .
\end{gathered}
$$




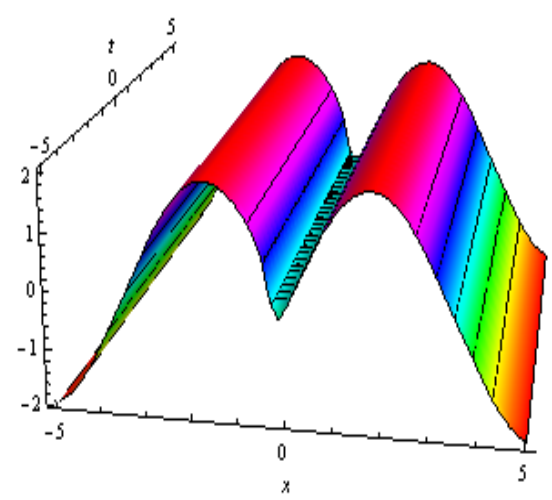

(a)

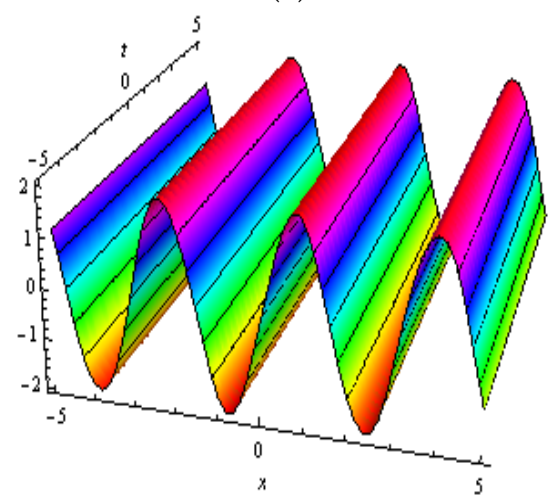

(d)

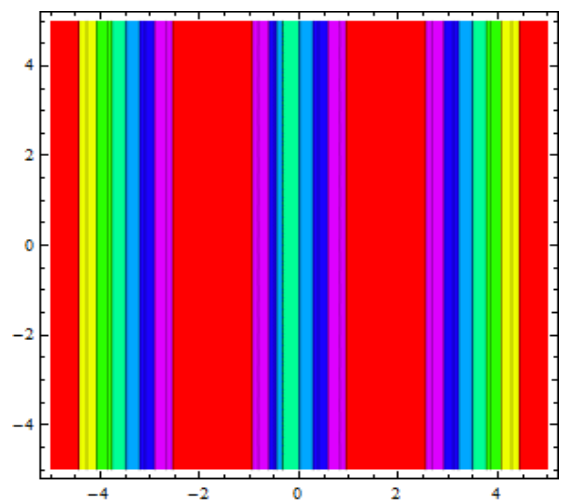

(b)

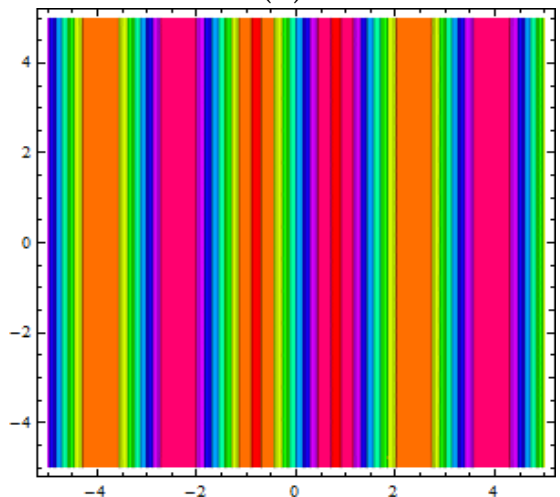

(e)

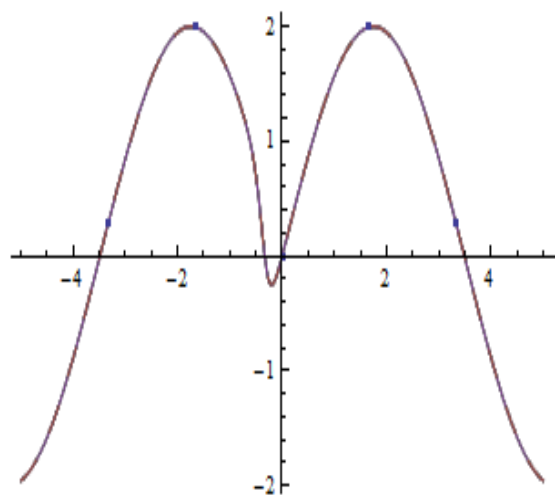

(c)

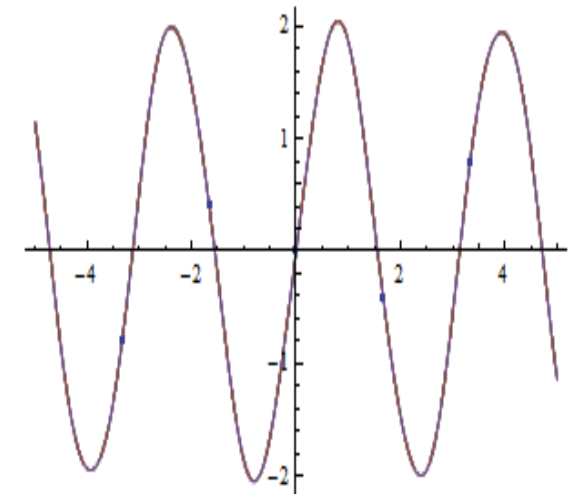

(f)

Figure 16. Showed three-dimensions in (a); contour in (b) and two-dimensions in (c), Graphical representation of $m_{63}(x, t)$ in Equation (62), for $b_{2}=-5, b_{3}=1, b_{6}=5$, $z_{1}=1, z_{2}=3, z_{3}=-0.5, \alpha=0.9$, respectively.

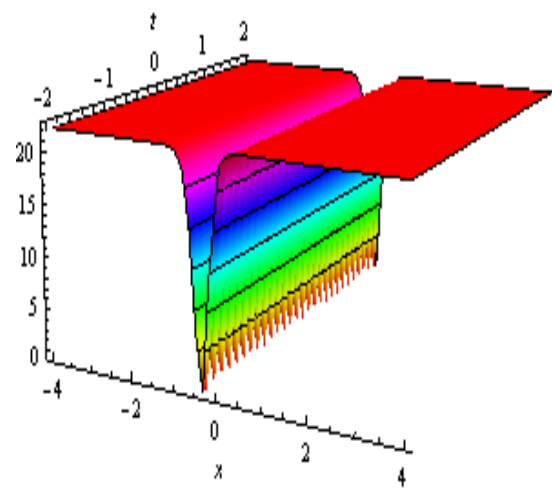

(a)

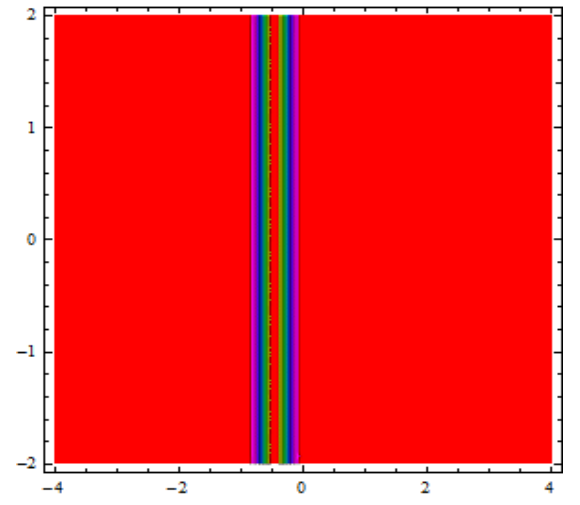

(b)

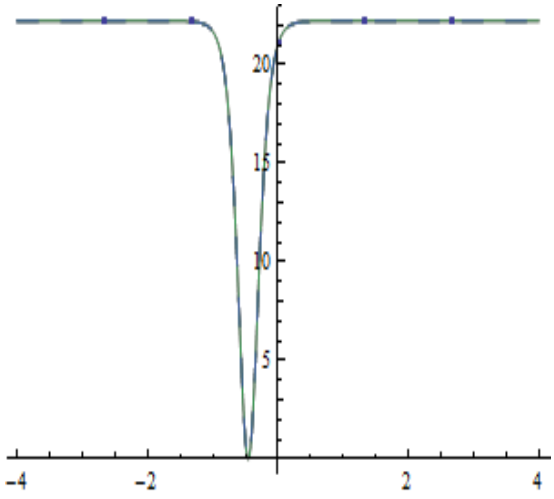

(c)

Figure 17. Cont. 


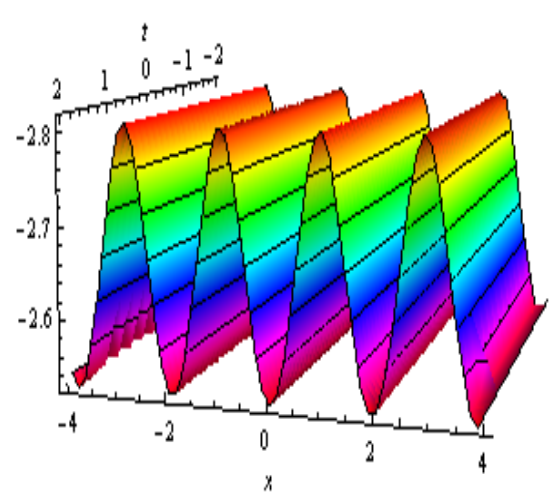

(d)

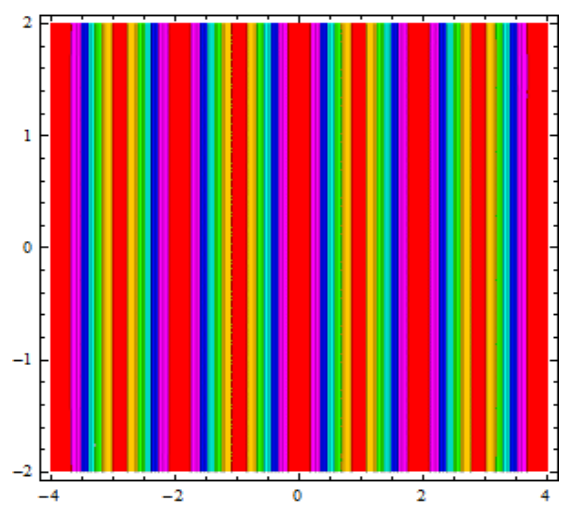

(e)

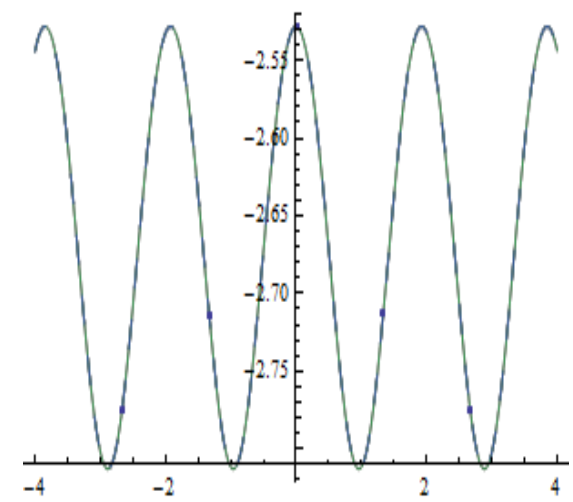

(f)

Figure 17. Represented three-dimensions in (a); contour in (b) and two-dimensions in (c), Graphical representation of $n_{64}(x, t)$ in Equation (62), for $b_{2}=-5, b_{3}=1, b_{6}=5$, $z_{1}=1, z_{2}=3, z_{3}=-0.5, \alpha=0.9$, respectively.

\section{Results and Discussion}

The study of new imposed solutions for the ion sound and Langmuir waves (ISLWs) has huge importance among scientists. Much of the work has been carried out on ISLWs, for example, Mohammed et al. constructed new traveling wave solutions for ISLWs by using He's semi-inverse and extended Jacobian elliptic function method [34]. Shakeel et al. studied new wave behaviors for ISLWs with the aid of modified exp-function approach [35]. Seadawy et al. used direct algebraic and auxiliary equation mapping to obtain the families of new exact traveling wave solutions for ISLWs [36]. Tripathy and Sahoo studied a variety of analytical solutions for ISLWs [37]. Seadawy et al. studied a variety of exact solutions with modified Kudraysov and hyperbolic-function scheme for ISLWs [38].

Here, we obtained a variety of analytical solutions with rational and trigonometric forms for ISLWs, in which some of them are represented graphically in 3D, contour, and 2D shapes. In Figures 1 and 2, we present M-shaped solutions for $m_{23}$ and $m_{25}$ with contour and 2D plots, respectively. In Figures 3-6, we see the interactional phenomena with M-shaped and one-kink for $m_{31}, n_{32}, m_{35}$, and $n_{36}$ at different values of the parameters. In these figures, we see M-shaped waves with multiple bright and dark solutions. In Figure 4, waves strongly increased their amplitude according to time. In Figures 7-9, we see the interactional phenomena with M-shaped and two-kink for $n_{38}, m_{39}$, and $n_{40}$. In Figure 7, multiple bright, dark, and M-size solitons appear. In Figures 8 and 9, large-sized dark and bright waves appear. Figures 10 and 11 represent the evolution of M-shaped and periodic waves for $m_{3}$ and $n_{4}$. Figures 12 and 13 represent the evolution of multiwaves solution for $m_{43}$ and $n_{44}$ at different values. In Figures 14 and 15, two solutions, $m_{51}$ and $n_{54}$, of homoclinic breather are presented graphically, and we also see the changes in graphs by varying the value of $a$. In Figures 16 and 17, we present periodic cross-kink solutions $m_{63}$ and $n_{44}$ graphically, and we also see the change in waves into bright and dark solutions by varying the value of $a$. As $\alpha \in(0,1]$, in all these solutions, we can see that when $\alpha=1$, $\sum_{s=0}^{\infty}\left(-\frac{\alpha}{1-\alpha}\right)^{s}$ does not converge.

\section{Conclusions}

In this work, we successfully derived some new analytic solutions for FISLWS with Atangana-Baleanu derivative. These exact solutions are derived in the form of bilinear, trigonometric, and exponential functions. As a result, new traveling wave solutions are gained in the form of rational, periodic, multiwaves, multi-kink, solitary waves, bright and dark solitons that are shown graphically in 3D, 2D, and contour structures. These solutions play an important role in different areas of physics, engineering, and other branches of sciences. 
Author Contributions: Methodology, S.T.R.R.; Resources, A.D.A.; Supervision, A.R.S.; Writing, S.A.O.B. All authors have read and agreed to the published version of the manuscript.

Funding: This research received no external funding.

Institutional Review Board Statement: Not applicable.

Informed Consent Statement: Not applicable.

Data Availability Statement: Not applicable.

Acknowledgments: This work was funded by the Deanship of Scientific Research at Jouf University under grant No (DSR-2021-03-03106).

Conflicts of Interest: The authors declare no conflict of interest.

\section{References}

1. Dalir, M.; Bashour, M. Applications of Fractional Calculus. Appl. Math. Sci. 2010, 4, 1021-1032.

2. Özkan, Y.S.; Seadawy, A.R.; Yaşar, E. Multi-wave, breather and interaction solutions to (3+1) dimensional Vakhnenko-Parkes equation arising at propagation of high-frequency waves in a relaxing medium. J. Taibah Univ. Sci. 2021, 15, 666-678. [CrossRef]

3. Lu, D.; Seadawy, A.R.; Iqbal, M. Mathematical physics via construction of traveling and solitary wave solutions of three coupled system of nonlinear partial differential equations and their applications. Results Phys. 2018, 11, 1161-1171. [CrossRef]

4. Seadawy, A.R.; Ali, A.; Albarakati, W.A. Analytical wave solutions of the (2+1)-dimensional first integro-differential KadomtsevPetviashivili hierarchy equation by using modified mathematical methods. Results Phys. 2019, 15, 102775. [CrossRef]

5. Seadawy, A.R.; Cheemaa, N. Propagation of nonlinear complex waves for the coupled nonlinear Schrödinger Equations in two core optical fibers. Phys. A Stat. Mech. Its Appl. 2019, 529, 121330. [CrossRef]

6. Kudryashov, N.A. The generalized Duffing oscillator. Commun. Nonlinear Sci. Numer. Simulat. 2021, 93, 105526. [CrossRef]

7. Kudryashov, N.A. Model of propagation pulses in an optical fiber with a new law of refractive index. Optik 2021, 248, 168160. [CrossRef]

8. Kudryashov, N.A. Solitary waves of the non-local Schrödinger equation with arbitrary refractive index. Optik 2021, $231,166443$. [CrossRef]

9. Kudryashov, N.A. Almost general solution of the reduced higher-order nonlinear Schrödinger equation. Optik 2021, 230, 166347. [CrossRef]

10. Kudryashov, N.A. Periodic and solitary waves in optical fiber Bragg gratings with dispersive reflectivity. Chin. J. Phys. 2020, 66, 401-405. [CrossRef]

11. Rizvi, S.T.R.; Seadawy, A.R.; Younis, M.; Ahmad, N.; Zaman, S. Optical dromions for perturbed fractional nonlinear Schrödinger equation with conformable derivatives. Opt. Quantum Electron. 2021, 53, 477. [CrossRef]

12. Younas, U.; Younis, M.; Seadawy, A.R.; Rizvi, S.T.R.; Althobaiti, S.; Sayed, S. Diverse exact solutions for modified nonlinear Schrödinger equation with conformable fractional derivative. Results Phys. 2021, 20, 103766. [CrossRef]

13. Mohammadi, H.; Kumar, S.; Rezapour, S.; Etemad, S. A theoretical study of the Caputo-Fabrizio fractional modeling for hearing loss due to Mumps virus with optimal control. Chaos Solitons Fractals 2021, 144, 110668 [CrossRef]

14. Nisar, K.S.; Jothimani, K.; Kaliraj, K.; Ravichandran, C. An analysis of controllability results for nonlinear Hilfer neutral fractional derivatives with non-dense domain. Chaos Solitons Fractals 2021, 146, 110915 [CrossRef]

15. Kaliraj, K.; Thilakraj, E.; Ravichandran, C.; Nisar, K.S. Controllability analysis for impulsive integro-differential equation via Atangana-Baleanu fractional derivative. Math. Methods Appl. Sci. 2020. [CrossRef]

16. Panda, S.K.; Ravichandran, C.; Hazarika, B. Ravichandran, Bipan Hazarika, Results on system of Atangana-Baleanu fractional order Willis aneurysm and nonlinear singularly perturbed boundary value problems. Chaos Solitons Fractals 2021, 142, 110390. [CrossRef]

17. Younas, U.; Seadawy, A.R.; Younis, M.; Rizvi, S. Construction of analytical wave solutions to the conformable fractional dynamical system of ion sound and Langmuir waves. Waves Random Complex Media 2020, 32, 1-19. [CrossRef]

18. Akram, U.; Seadawy, A.R.; Rizvi, S.T.R.; Younis, M.; Althobaiti, S.; Sayed, S. Traveling waves solutions for the fractional Wazwaz Benjamin Bona Mahony model in arising shallow water waves. Results Phys. 2021, 20, 103725. [CrossRef]

19. Dokuyucua, M.A. Caputo and Atangana-Baleanu-Caputo Fractional Derivative Applied to Garden Equation. Turk. J. Sci. 2020, 5, $1-7$.

20. Seadawy, A.R. Modulation instability analysis for the generalized derivative higher order nonlinear Schrödinger equation and its the bright and dark soliton solutions. J. Electromagn. Waves Appl. 2017, 31, 1353-1362 [CrossRef]

21. Zhang, S.; Zong, Q.A.; Liu, D.; Gao, Q. A generalized exp-function method for fractional Riccati differential equations. Commun. Fract. Calc. 2010, 1, 48-51.

22. Seadawy, A.R.; Tariq, K.U. On some novel solitons to the generalized $(1+1)$-dimensional unstable spacetime fractional nonlinear Schrödinger model emerging in the optical fibers. Opt. Quantum Electron. 2021, 53, 1-16. [CrossRef]

23. Chen, C.; Jiang, Y.; Wang, Z.; Wu, J. Dynamical behavior and exact solutions for time-fractional nonlinear Schrödinger equation with parabolic law nonlinearity. Optik 2020, 222, 165331. [CrossRef] 
24. Khan, S.A.; Shah, K.; Kumam, P.; Seadawy, A.; Zaman, G.; Shah, Z. Study of mathematical model of Hepatitis B under Caputo-Fabrizo derivative. AIMS Math. 2021, 6, 195-209. [CrossRef]

25. Seadawy, A.R.; Bilal, M.; Younis, M.; Rizvi, S.; Althobaiti, S.; Makhlouf, M. Analytical mathematical approaches for the doublechain model of DNA by a novel computational technique. Chaos Solitons Fractals 2021, 144, 110669. [CrossRef]

26. Farah, N.; Seadawy, A.R.; Ahmad, S.; Rizvi, S.T.R.; Younis, M. Interaction properties of soliton molecules and Painleve analysis for nano bioelectronics transmission model. Opt. Quantum Electron. 2020, 52, 1-15. [CrossRef]

27. Helal, M.A.; Seadawy, A.R. Variational method for the derivative nonlinear Schrödinger equation with computational applications. Phys. Scr. 2009, 80, 350-360. [CrossRef]

28. Gaber, A.A.; Aljohani, A.F.; Ebaid, A.; Machado, J.T. The generalized Kudryashov method for nonlinear space-time fractional partial differential equations of Burgers type. Nonlinear Dyn. 2019, 95, 361-368. [CrossRef]

29. Ghaffar, A.; Ali, A.; Ahmed, S.; Akram, S.; Junjua, M.-U.-D.; Baleanu, D.; Nisar, K.S. A novel analytical technique to obtain the solitary solutions for nonlinear evolution equation of fractional order. Adv. Differ. Equ. 2020, 2020, 308. [CrossRef]

30. Rizvi, S.T.R.; Ali, K.; Ahmad, M. Optical solitons for Biswas-Milovic equation by new extended auxiliary equation method. Optik 2020, 204, 164181. [CrossRef]

31. Ahmed, I.; Seadawy, A.R.; Lu, D. M-shaped rational solitons and their interaction with kink waves in the Fokas-Lenells equation. Phys. Scr. 2019, 94, 055205. [CrossRef]

32. Ahmed, I.; Seadawy, A.R.; Lu, D. Kinky breathers, W-shaped and multi-peak solitons interaction in $(2+1)$-dimensional nonlinear Schrödinger equation with Kerr law of nonlinearity. Eur. Phys. J. Plus 2019, 134, 120. [CrossRef]

33. Ma, H.; Zhang, C.; Deng, A. New Periodic Wave, Cross-Kink Wave, Breather, and the Interaction Phenomenon for the (2+1)Dimensional Sharmo-Tasso-Olver Equation. Complexity 2020, 2020, 4270906. [CrossRef]

34. Mohammed, W.W.; Abdelrahman, M.A.E.; Inc, M.; Hamza, A.E.; Akinlar, M.A. Soliton solutions for system of ion sound and Langmuir waves. Opt. Quantum Electron. 2020, 52, 1-10. [CrossRef]

35. Shakeel, M.; Iqbal, M.A.; Din, Q.; Hassan, Q.M.; Ayub, K. New exact solutions for coupled nonlinear system of ion sound and Langmuir waves. Indian J. Phys. 2019, 94, 885-894. [CrossRef]

36. Seadawy, A.R.; Lu, D.; Iqbal, M. Application of mathematical methods on the system of dynamical equations for the ion sound and Langmuir waves. Pramana 2019, 93, 1-12. [CrossRef]

37. Tripathy, A.; Sahoo, S. Exact solutions for the ion sound Langmuir wave model by using two novel analytical methods. Results Phys. 2020, 19, 103494. [CrossRef]

38. Seadawy, A.R.; Kumar, D.; Hosseini, K.; Samadani, F. The system of equations for the ion sound and Langmuir waves and its new exact solutions. Results Phys. 2018, 9, 1631-1634. [CrossRef] 